\title{
Numerical Scheme for Finding Roots of Interval-Valued Fuzzy Nonlinear Equation with Application in Optimization
}

\author{
Ahmed Elmoasry $\mathbb{D}^{1},{ }^{1}$ Mudassir Shams $\mathbb{D}^{2},{ }^{2}$ Naveed Yaqoob $\mathbb{D}^{2},{ }^{2}$ Nasreen Kausar $\mathbb{D}{ }^{3}$ \\ Yaé Ulrich Gaba $\mathbb{D}^{4,5,6}$ and Naila Rafiq $\left.{ }^{7}\right)^{7}$ \\ ${ }^{1}$ Department of Mathematics, College of Science Al-Zulfi, Majmaah University, Al-Zulf, Saudi Arabia \\ ${ }^{2}$ Department of Mathematics and Statistics, Riphah International University I-14, Islamabad 44000, Pakistan \\ ${ }^{3}$ Department of Mathematics, Yildiz Technical University, Faculty of Arts and Science, Esenler, 34210 Istanbul, Turkey \\ ${ }^{4}$ Quantum Leap Africa (QLA), AIMS Rwanda Centre, Remera Sector KN 3, Kigali, Rwanda \\ ${ }^{5}$ Institut de Mathématiques et de Sciences Physiques (IMSP/UAC), Laboratoire de Topologie Fondamentale, Computationnelle et \\ Leurs Applications (Lab-ToFoCApp), BP 613, Porto-Novo, Benin \\ ${ }^{6}$ African Center for Advanced Studies, P.O. Box 4477, Yaounde, Cameroon \\ ${ }^{7}$ Department of Mathematics, NUML, Islamabad, Pakistan
}

Correspondence should be addressed to Yaé Ulrich Gaba; yaeulrich.gaba@gmail.com

Received 17 June 2021; Accepted 16 July 2021; Published 6 September 2021

Academic Editor: Mohsan Raza

Copyright (c) 2021 Ahmed Elmoasry et al. This is an open access article distributed under the Creative Commons Attribution License, which permits unrestricted use, distribution, and reproduction in any medium, provided the original work is properly cited.

In this research article, we propose efficient numerical iterative methods for estimating roots of interval-valued trapezoidal fuzzy nonlinear equations. Convergence analysis proves that the order of convergence of numerical schemes is 3 . Some real-life applications are considered from optimization as numerical test problems which contain interval-valued trapezoidal fuzzy quantities in parametric form. Numerical illustrations are given to show the dominance efficiency of the newly constructed iterative schemes as compared to existing methods in literature.

\section{Introduction}

One of the ancient problems of science and engineering in general and in mathematics is to approximate roots of a nonlinear equation. The nonlinear equations play a major role in the field of engineering, mathematics, physics, chemistry, economics, medicines finance, and in optimization. Many times the particular realization of such type of nonlinear problems involves imprecise and nonprobabilistic uncertainties in the parameter, where the approximations are known due to expert knowledge or due to some experimental data. Due to these reasons, several real-world applications contain vagueness and uncertainties. Therefore, in most of real-world problems, the parameter involved in the system or variables of the nonlinear functions are presented by a fuzzy number or interval-valued trapezoidal fuzzy number. The concept of fuzzy numbers and arithmetic operation with fuzzy numbers were first introduced and investigated in [1-8]. Hence, it is necessary to approximate the roots of fuzzy nonlinear equation.

$$
F(r)=c
$$

The standard analytical technique like the Buckley and Qu method [9-12] is not suitable for solving the equations like

$$
\begin{gathered}
a r^{6}+b r^{4}-c r^{3}+d r-e=f, r+\cos (r)=g, r \ln (r)+e^{r} \\
-\frac{1}{1+r^{2}}+\tan (r)=h,
\end{gathered}
$$

where $a, b, c, d, e, f, g$, and $h$ are fuzzy numbers and $r$ is a fuzzy variable. 


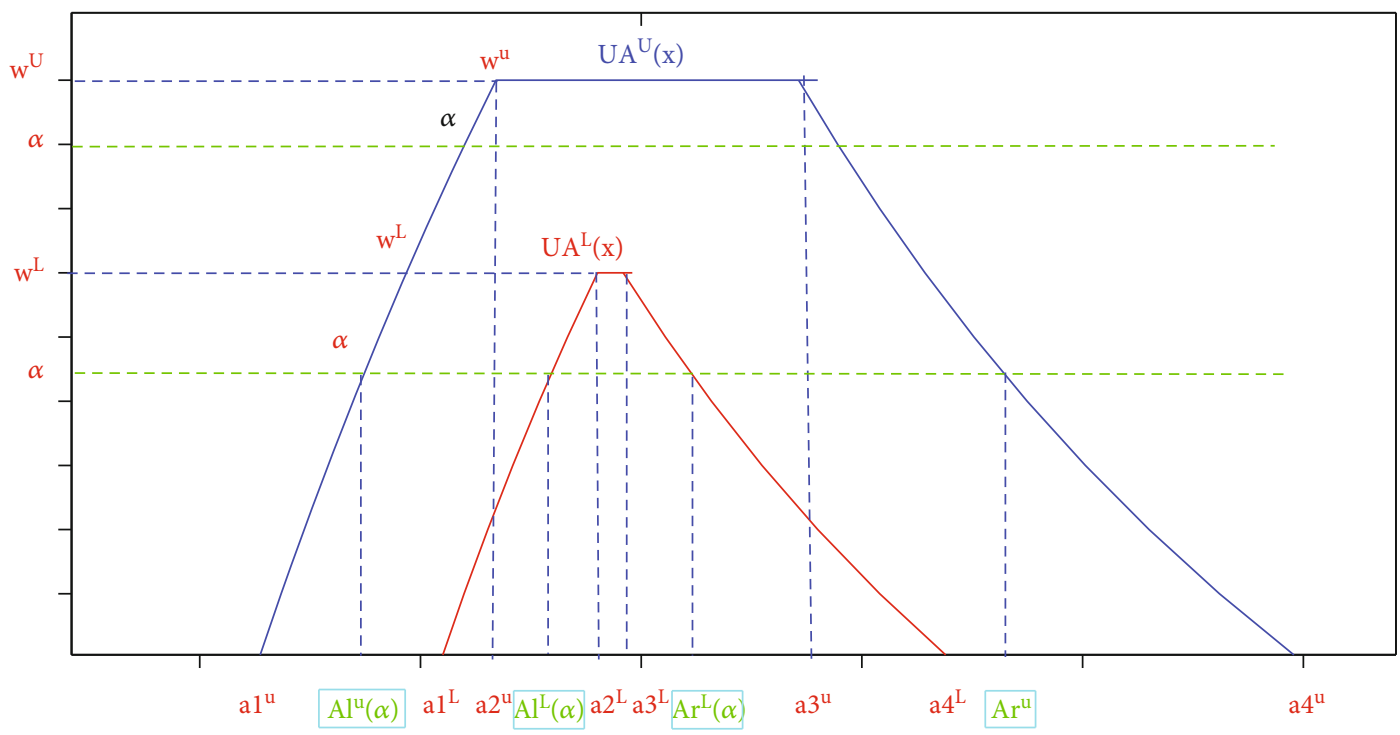

Figure 1: Alpha-cut level of interval-valued trapezoidal fuzzy number A.

We therefore look towards numerical iterative schemes which approximate the roots of fuzzy nonlinear equations. To approximate roots of fuzzy nonlinear equations, Abbsbandy and Asady [13] used Newton's method, Allahviranloo and Asari [14] used the Newton-Raphson method, Mosleh [15] used the Adomian decomposition method, and Ibrahim et al. give the Levenberg-Marquest method (see also [16-23]).

This research article is aimed at proposing efficient higher order iterative method as compared to well-known classical method, such as the Newton-Raphson method. Numerical test results, CPU time, and log of residual show the dominance efficiency of our newly constructed method over the classical Newton's method.

This paper is organized in five sections. In Section 2, we recall some fundamental results of interval-valued trapezoidal fuzzy numbers. In Section 3, we propose numerical iterative scheme for approximating roots of interval-valued trapezoidal fuzzy nonlinear equations and its convergence analysis. In Section 4, we illustrate some real-world applications from optimization as numerical test examples to show the performance and efficiency of the constructed method and conclusions in the last section. Section 5 is a conclusion section.

\section{Preliminaries}

Definition 1. A fuzzy number is a fuzzy set like $r \quad: \mathrm{R} \longrightarrow$ $I=[0,1]$ which satisfies [24-27].

(1) $r$ is upper semicontinuous

(2) $r(a)=0$ outside some interval $\left[a_{1}, a_{2}\right]$

(3) There are real numbers $b_{1}, b_{2}$ such that $a_{1} \leq b_{1} \leq b_{2}$ $\leq a_{2}$ and

(i) $r(a)$ is monotonic increasing on $\left[a_{1}, b_{1}\right]$

(ii) $r(a)$ is monotonic decreasing on $\left[b_{2}, a_{2}\right]$ (iii) $r(a)=1$, for $b_{1} \leq a \leq b_{2}$

We denote by $E$, the set of all fuzzy numbers. An equivalent parametric form is also given in [19] as follows.

Definition 2 [28]. A fuzzy number $r$ in parametric form is a pair $\left(r^{L}, r^{U}\right)$ of function $r^{L}(\tau), r^{U}(\tau), 0 \leq \tau \leq 1$, which satisfies the following requirements:

(1) $r^{L}(\tau)$ is a bounded monotonic increasing left continuous function

(2) $r^{U}(\tau)$ is a bounded monotonic decreasing left continuous function

(3) $r^{L}(\tau) \leq r^{U}(\tau), 0 \leq \tau \leq 1$

A popular fuzzy number is the generalized intervalvalued trapezoidal fuzzy number $A$, denoted by $A=\left(a_{1}, a_{2}\right.$, $\left.a_{3}, a_{4} ; \widehat{w}\right), 0<\widehat{w}<1$, a fuzzy number with membership function as follows:

$$
A(r)= \begin{cases}\widehat{w} \frac{r-a_{1}}{a_{2}-a_{1}} & \text { if } a_{1}<r<a_{2}, \\ \widehat{w} & \text { if } a_{2} \leq r \leq a_{3} \\ \widehat{w} \frac{a_{4}-r}{a_{4}-a_{3}} & a_{3}<r<a_{4}, \\ 0 & \text { otherwise. }\end{cases}
$$

Assume $F_{\mathrm{TN}}(\widehat{w})$ be the family of all $\widehat{w}$-trapezoidal fuzzy number, i.e.

$$
F_{\mathrm{TN}}(\widehat{w})=\left\{\begin{array}{c}
A=\left(a_{1}, a_{2}, a_{3}, a_{4} ; \widehat{w}\right), a_{1} \leq a_{2} \leq a_{3} \leq a_{4} ; \\
0<\widehat{w}<1
\end{array}\right\} .
$$




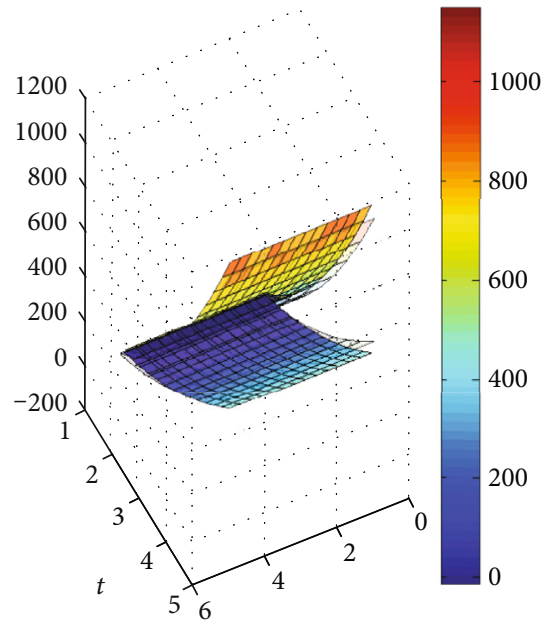

Analytical solution of example 1

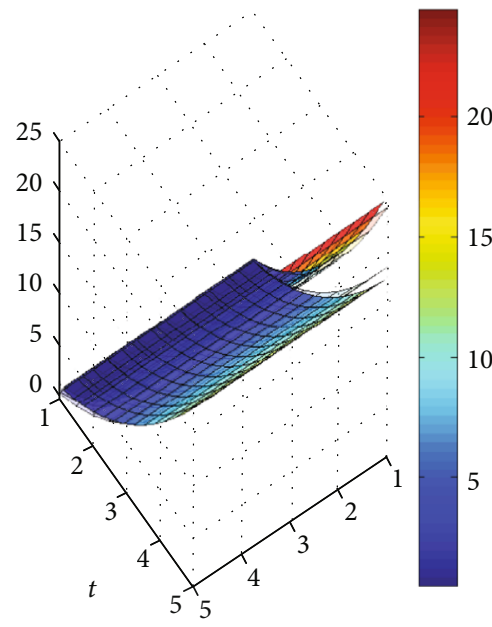

Analytical solution of example 2

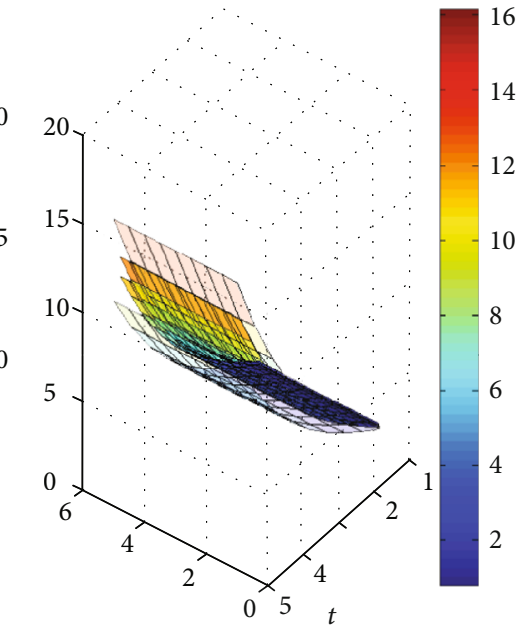

Analytical solution of example 3

FIgURE 2: Analytical solution of Examples 1-3.

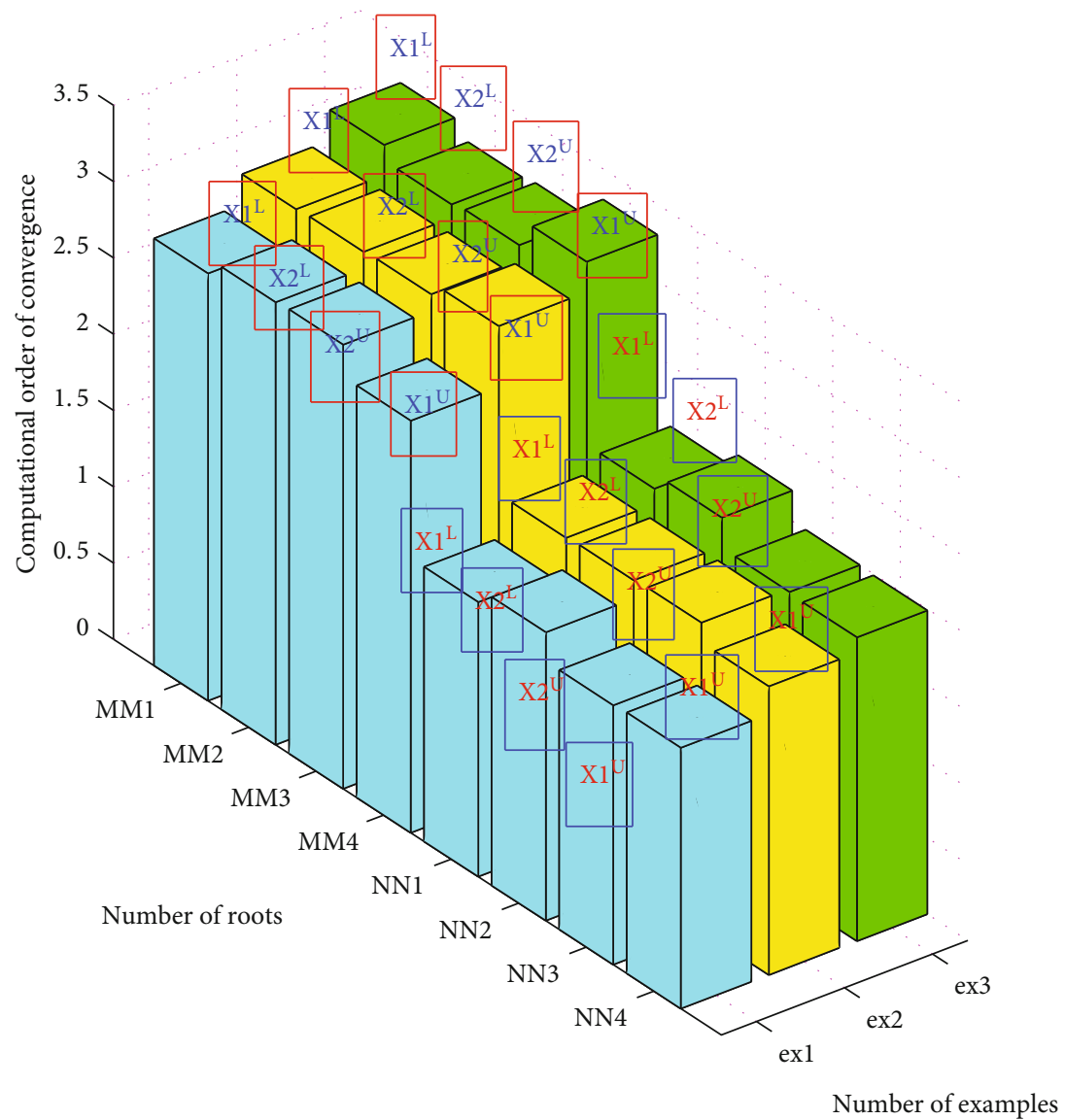

FIGURE 3: Computational order of convergence. 


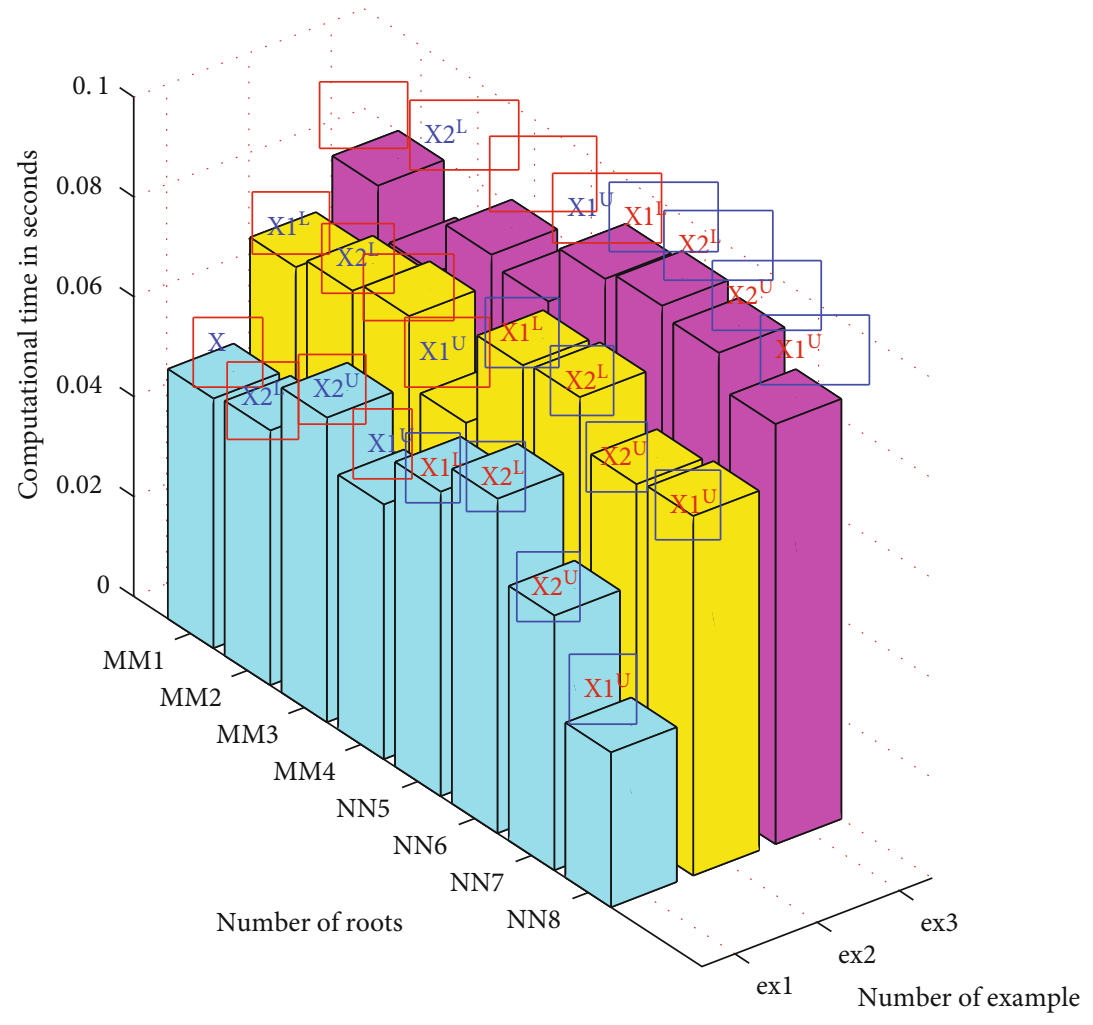

Figure 4: Computational time in seconds.
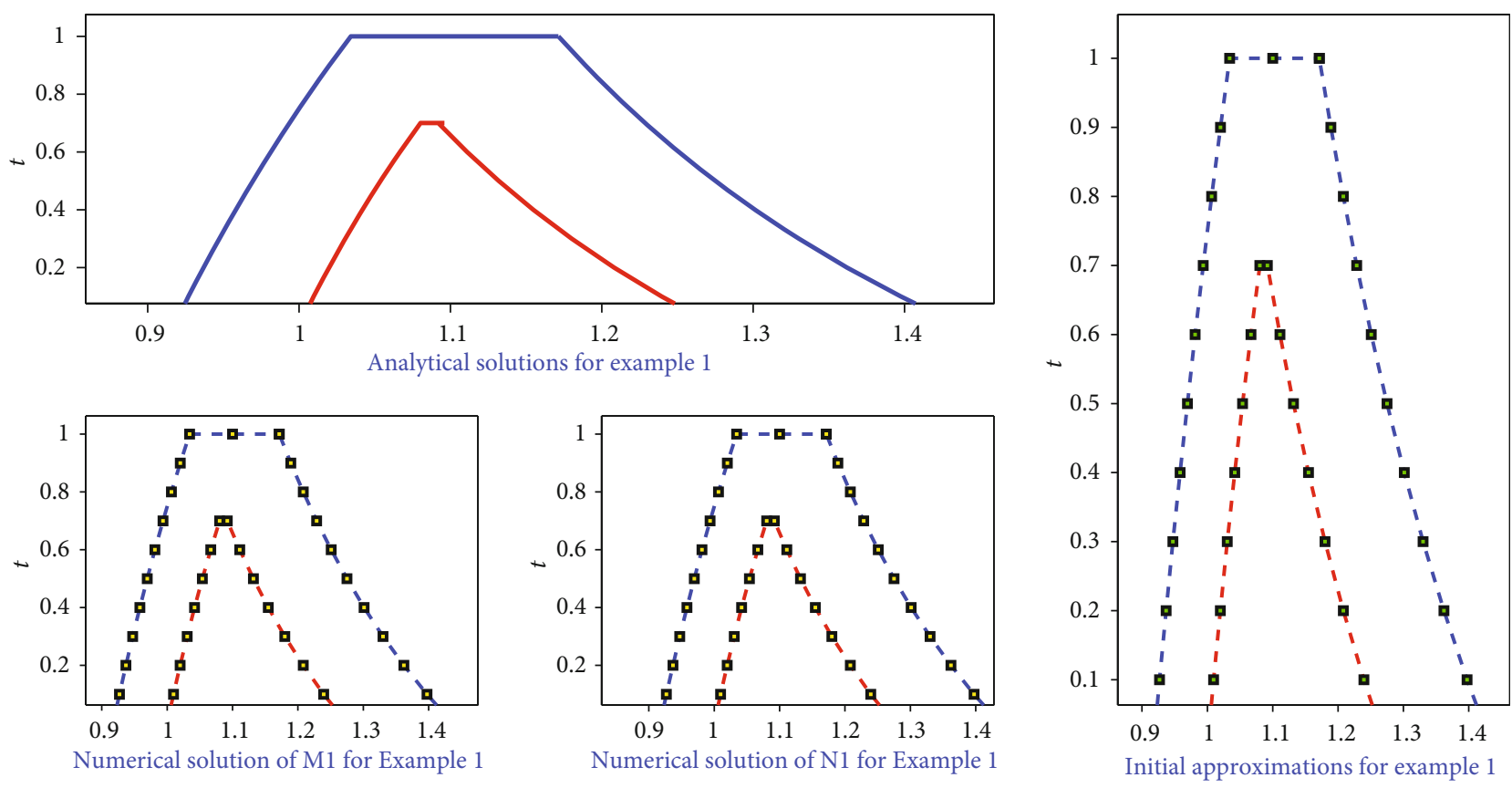

FIGURE 5: Initial guessed values, analytical, and numerical approximate solution. 

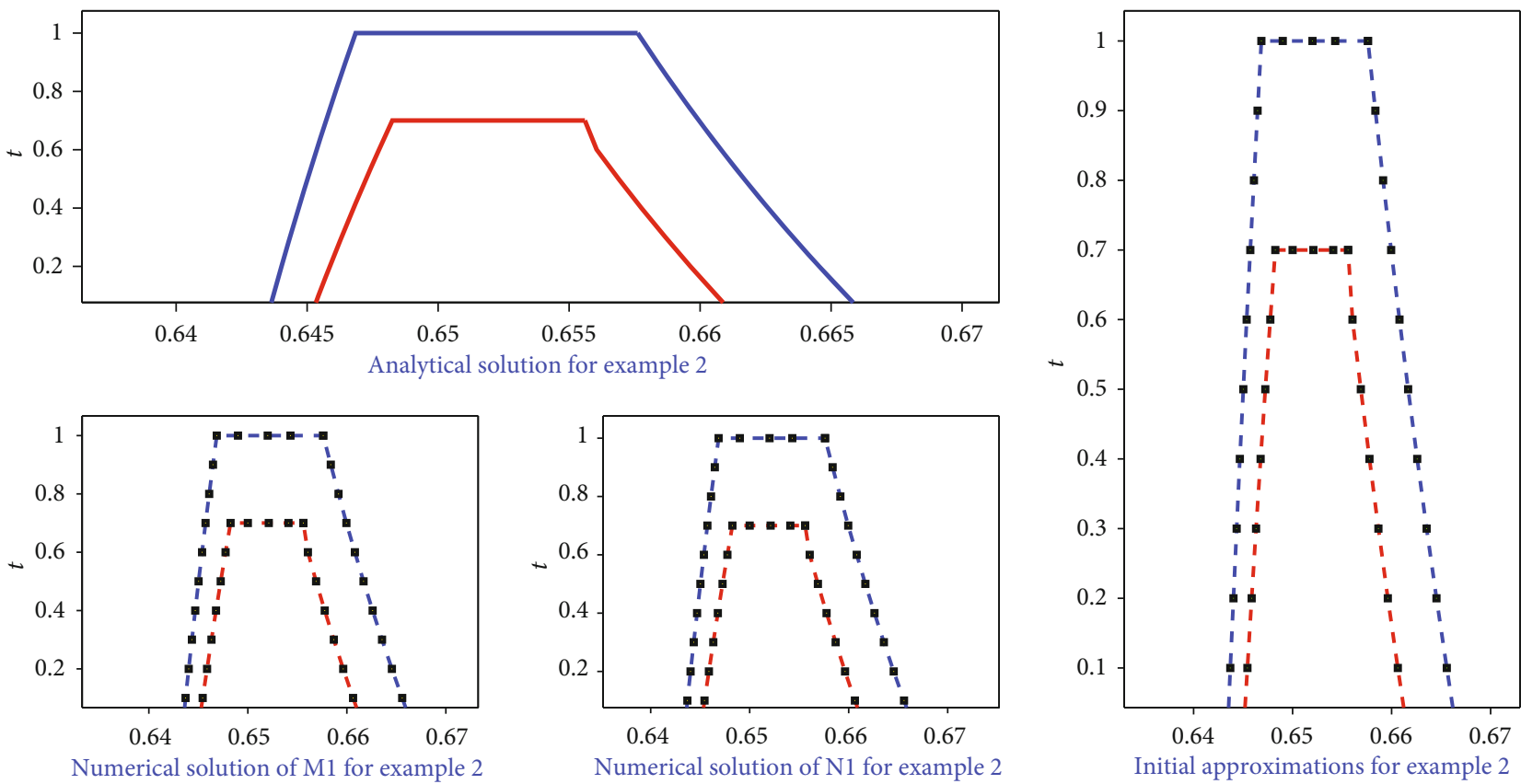

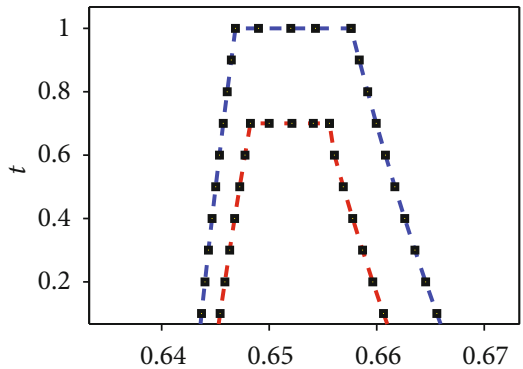

Numerical solution of M1 for example 2

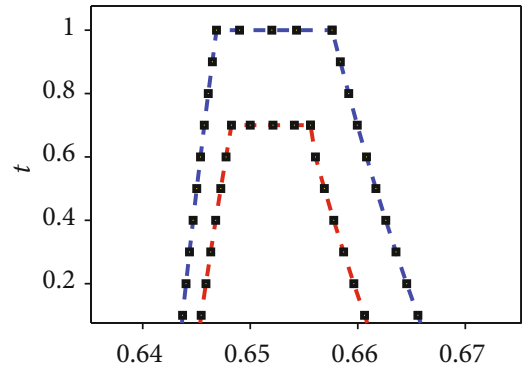

Numerical solution of $\mathrm{N} 1$ for example 2

Initial approximations for example 2

Figure 6: Initial guessed values, analytical, and numerical approximate solution.
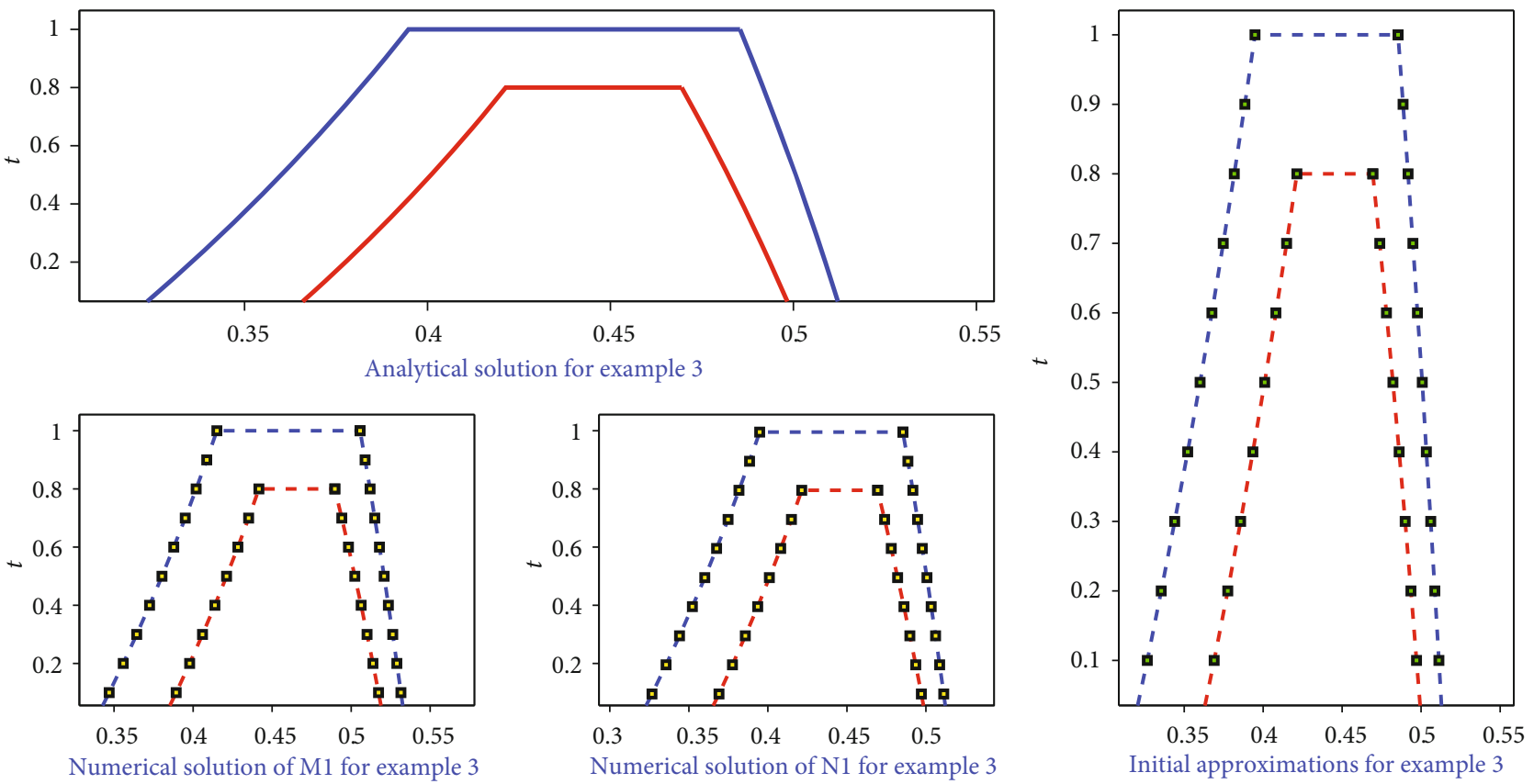

Initial approximations for example 3

Figure 7: Initial guessed values, analytical, and numerical approximate solution. 


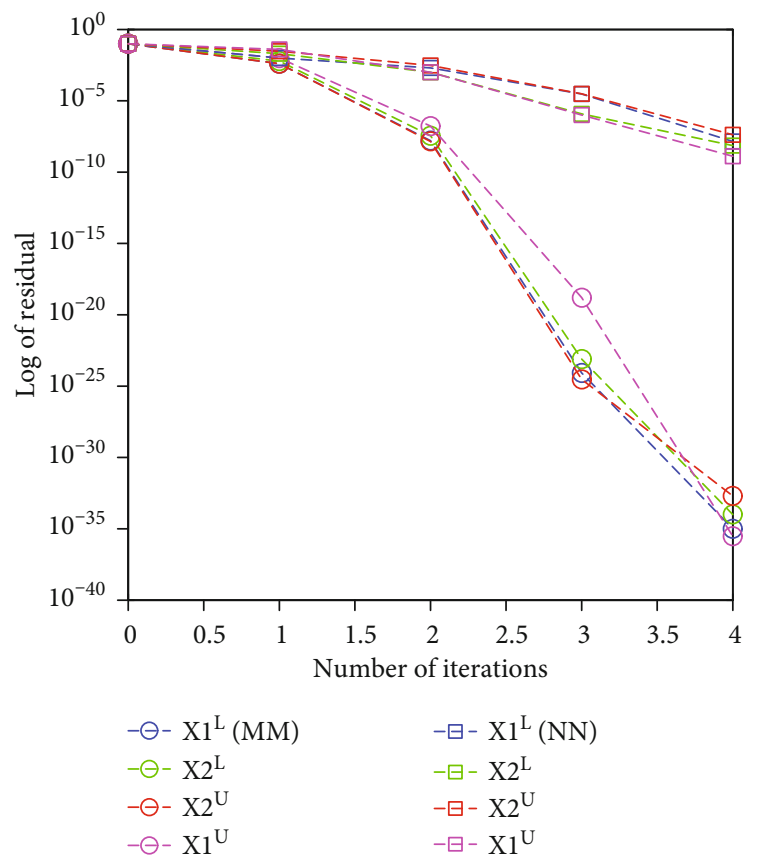

(a)

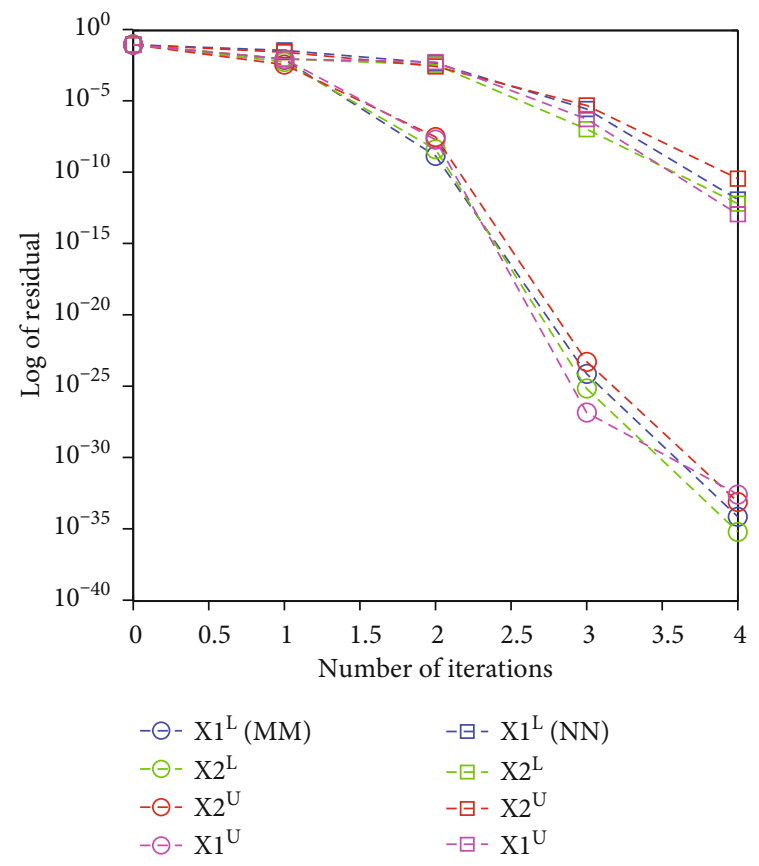

(b)

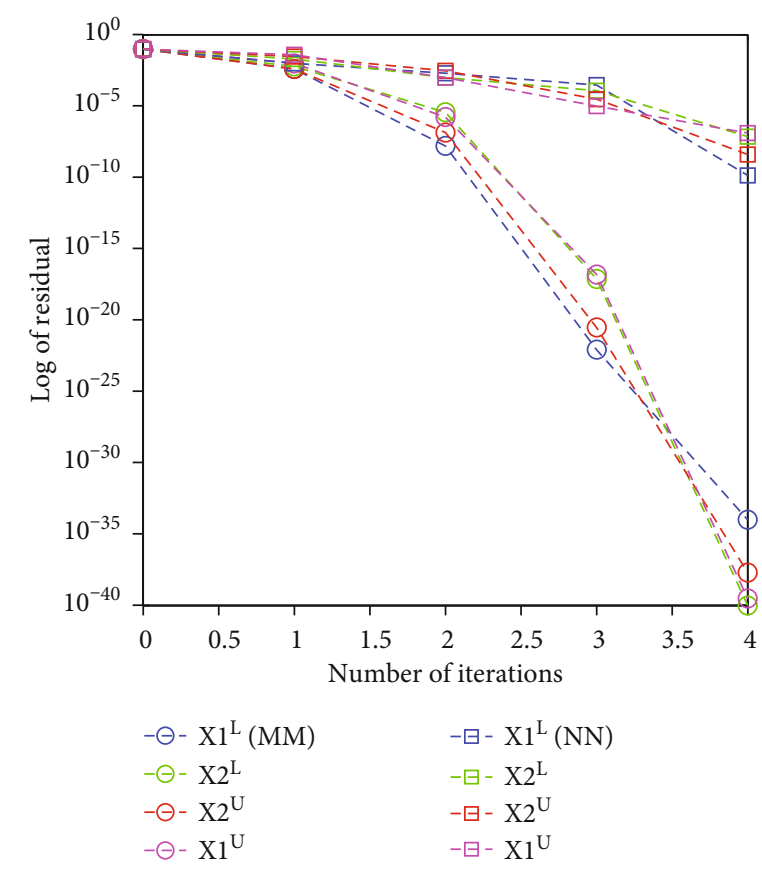

(c)

Figure 8: Error graph of iterative methods MM and NN. 


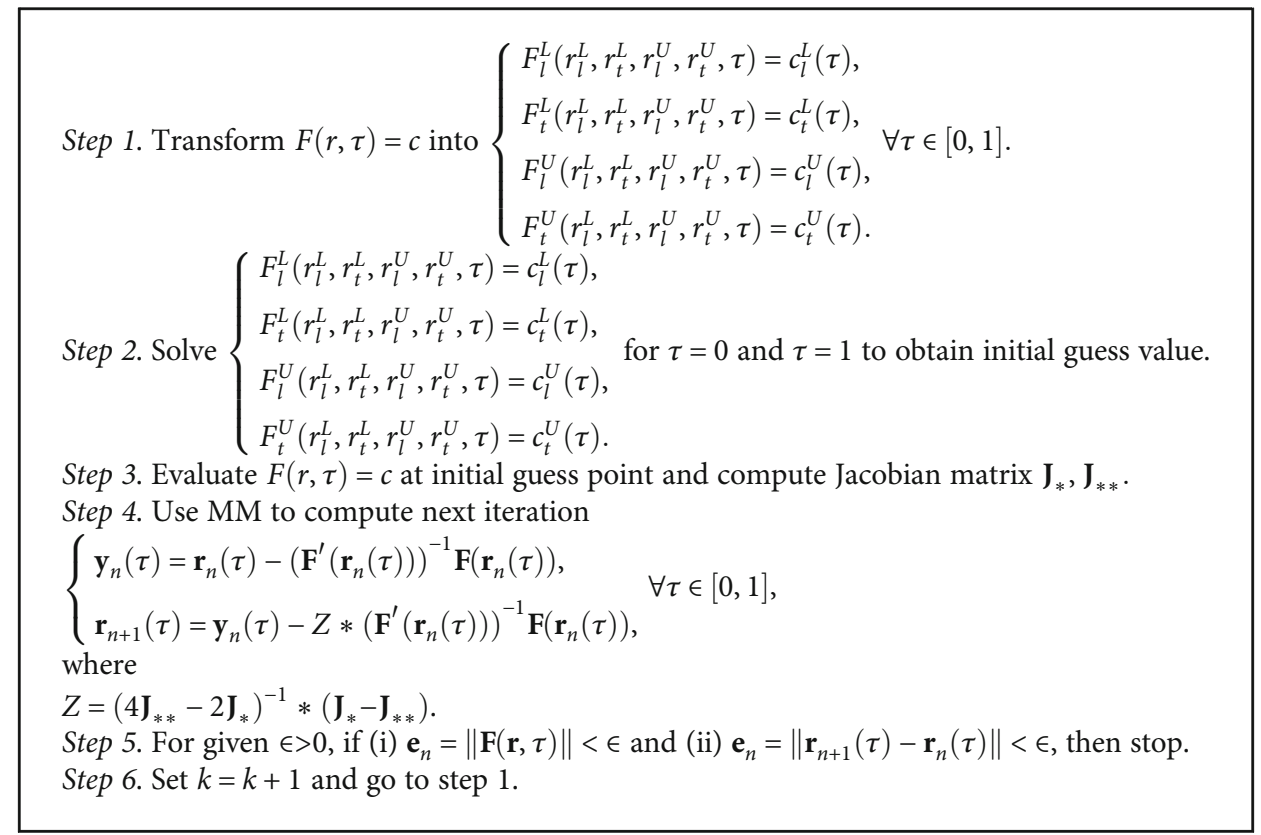

Algorithm 1: (MM method).

Definition 3 [29]. Let $A^{L} \in F_{\mathrm{TN}}\left(w \wedge^{L}\right)$ and $A^{U} \in F_{\mathrm{TN}}\left(w \wedge^{U}\right) A$ level $\left(w \wedge^{L}, w \wedge^{U}\right)$ - interval-valued trapezoidal fuzzy number $A$, denoted by

$$
\mathbf{A}=\left[A^{L}, A^{U}\right]=\left\langle\left(a_{1}^{L}, a_{2}^{L}, a_{3}^{L}, a_{4}^{L} ; w \wedge^{L}\right),\left(a_{1}^{U}, a_{2}^{U}, a_{3}^{U}, a_{4}^{U} ; w \wedge^{U}\right)\right\rangle,
$$

is an interval-valued fuzzy number on set $\mathrm{R}$ with

$$
\begin{aligned}
& A^{L}(r)=\left\{\begin{array}{ll}
w \wedge^{L} \frac{r-a_{1}^{L}}{a_{2}^{L}-a_{1}^{L}} & \text { if } a_{1}^{L}<r<a_{2}^{L}, \\
w \wedge^{L} & \text { if } a_{2}^{L} \leq r \leq a_{3}^{L}, \\
w \wedge^{L} \frac{a_{4}^{L}-r}{a_{4}^{L}-a_{3}^{L}} & a_{3}^{L}<r<a_{4}^{L}, \\
0 & \text { otherwise, }
\end{array}\right. \text { (lower trapezoidal fuzzy number) } \\
& A^{U}(r)= \begin{cases}w \wedge^{U} \frac{r-a_{1}^{U}}{a_{2}^{U}-a_{1}^{U}} & \text { if } a_{1}^{U}<r<a_{2}^{U}, \\
w \wedge^{U} & \text { if } a_{2}^{U} \leq r \leq a_{3}^{U}, \\
w \wedge^{U} \frac{a_{4}^{U}-r}{a_{4}^{U}-a_{3}^{U}} & a_{3}^{U}<r<a_{4}^{U}, \\
0 & \text { otherwise. }\end{cases}
\end{aligned}
$$

where $a_{1}^{L} \leq a_{2}^{L} \leq a_{3}^{L} \leq a_{4}^{L}, a_{1}^{U} \leq a_{2}^{U} \leq a_{3}^{U} \leq a_{4}^{U}, 0 \leq w \wedge^{L} \leq w \wedge^{U}$ $\leq 1, a_{1}^{U} \leq a_{1}^{L}$, and $a_{1}^{L} \leq a_{4}^{U}$. This interval-valued trapezoidal fuzzy number is shown in Figure 1. Moreover, $A^{L}(r) \leq A^{U}($ $r)$, which means the grade of membership $r \in \mathbf{A}=\left[A^{L}(r)\right.$, $\left.A^{U}(r)\right]$, and the latest and greatest grade of membership at $r$ are $A^{L}(r)$ and $A^{U}(r)$, respectively. We therefore denote the family of all interval-valued trapezoidal fuzzy number
TABle 1

\begin{tabular}{lcccc}
\hline$\tau$ & \multicolumn{2}{c}{$\mathrm{MM}$} & \multicolumn{2}{c}{$\mathrm{NN}$} \\
\hline 0.0 & $\left\|\mathbf{r}_{n+1}-\mathbf{r}_{n}\right\|$ & $\left\|\mathbf{F}\left(\mathbf{r}_{n}\right)\right\|$ & $\left\|\mathbf{r}_{n+1}-\mathbf{r}_{n}\right\|$ & $\left\|\mathbf{F}\left(\mathbf{r}_{n}\right)\right\|$ \\
0.1 & $3.1 e-47$ & $1.2 e-36$ & $4.1 e-10$ & $1.4 e-8$ \\
0.2 & $1.1 e-43$ & $3.1 e-32$ & $3.1 e-10$ & $1.1 e-8$ \\
0.3 & $1.4 e-45$ & $1.1 e-32$ & $0.1 e-20$ & $8.2 e-7$ \\
0.4 & $5.6 e-46$ & $3.4 e-34$ & $1.7 e-10$ & $7.2 e-9$ \\
0.5 & $1.3 e-41$ & $1.4 e-32$ & $1.5 e-16$ & $5.5 e-9$ \\
0.6 & $4.4 e-41$ & $1.7 e-32$ & $7.2 e-11$ & $4.1 e-9$ \\
0.7 & $6.1 e-49$ & $7.1 e-37$ & $3.2 e-11$ & $3.1 e-9$ \\
0.8 & $8.1 e-47$ & $9.1 e-33$ & $4.1 e-10$ & $6.2 e-9$ \\
0.9 & $1.3 e-45$ & $4.1 e-32$ & $6.3 e-11$ & $7.5 e-9$ \\
1 & $1.6 e-46$ & $6.4 e-33$ & $7.8 e-11$ & $8.1 e-9$ \\
\hline
\end{tabular}

by $F\left(w \wedge^{L}, w \wedge^{U}\right)=\mathbf{A}=\left[A^{L}(r), A^{U}(r)\right]$, i.e.,

$$
\begin{gathered}
F\left(w \wedge^{L}, w \wedge^{U}\right)=\mathbf{A}=\left[A^{L}(r), A^{U}(r)\right]=\left\{\left\langle\left(a_{1}^{L}, a_{2}^{L}, a_{3}^{L}, a_{4}^{L} ; w \wedge^{L}\right),\right.\right. \\
\left.\left(a_{1}^{U}, a_{2}^{U}, a_{3}^{U}, a_{4}^{U} ; w \wedge^{U}\right)\right\rangle: a_{1}^{U} \leq a_{1}^{L} ; a_{4}^{L} \leq a_{4}^{U} \\
A^{L}(r) \in F_{\mathrm{TN}}\left(w \wedge^{L}\right), A^{U}(r) \in F_{\mathrm{TN}}\left(w \wedge^{U}\right), 0 \leq w \wedge^{L} \leq w \wedge^{U} \leq 1 .
\end{gathered}
$$

Definition 4 [29]. A $\left(w \wedge^{L}, w \wedge^{U}\right)$ is said to be nonnegative $F$ $\left(w \wedge^{L}, w \wedge^{U}\right)$ iff $a_{1}^{U} \geq 0$ and denoted by $F^{+}\left(w \wedge^{L}, w \wedge^{U}\right)$.

Definition 5 [30]. Two $\left(w \wedge^{L}, w \wedge^{U}\right)$ - interval-valued trapezoidal fuzzy numbers.

$A=\left\langle\left(a_{1}^{L}, a_{2}^{L}, a_{3}^{L}, a_{4}^{L} ; w \wedge^{L}\right),\left(a_{1}^{U}, a_{2}^{U}, a_{3}^{U}, a_{4}^{U} ; w \wedge^{U}\right)\right\rangle$ and $B$ $=\left\langle\left(b_{1}^{L}, b_{2}^{L}, b_{3}^{L}, b_{4}^{L} ; w \wedge^{L}\right),\left(b_{1}^{U}, b_{2}^{U}, b_{3}^{U}, b_{4}^{U} ; w \wedge^{U}\right)\right\rangle$ are said to 
TABle 2: Analytical solution for Example 1.

\begin{tabular}{lccccccccccc}
\hline$\tau$ & 0.0 & 0.1 & 0.2 & 0.3 & 0.4 & 0.5 & 0.6 & 0.7 & 0.8 & 0.9 & 1.0 \\
$r_{l}^{U}$ & 0.9172 & 0.9268 & 0.9368 & 0.9473 & 0.9581 & 0.9694 & 0.9812 & 0.9936 & 1.0065 & 1.0200 & 1.0342 \\
$r_{l}^{L}$ & 1.0000 & 1.0096 & 1.0197 & 1.0304 & 1.0418 & 1.0538 & 1.0666 & 1.0802 & 1.0947 & 1.1102 & 1.1269 \\
$r_{t}^{L}$ & 1.2749 & 1.2394 & 1.2080 & 1.1800 & 1.1547 & 1.1318 & 1.1109 & 1.0918 & 1.0743 & 1.0581 & 1.0430 \\
$r_{t}^{U}$ & 1.4371 & 1.3974 & 1.3620 & 1.3301 & 1.3012 & 1.2749 & 1.2507 & 1.2285 & 1.2080 & 1.1890 & 1.1712 \\
\hline
\end{tabular}

be equal iff $A=B$, i.e., $a_{i}^{L}=b_{i}^{L}$ and $a_{i}^{U}=b_{i}^{U}$ for all $i=1,2,3,4$.

Definition 6. [30]. Extend addition, scalar multiplication, and extend multiplication in $\left(w \wedge^{L}, w \wedge^{U}\right)$ interval-valued trapezoidal fuzzy number are defined as if $A=\left\langle\left(a_{1}^{L}, a_{2}^{L}, a_{3}^{L}, a_{4}^{L}\right.\right.$; $\left.\left.w \wedge^{L}\right),\left(a_{1}^{U}, a_{2}^{U}, a_{3}^{U}, a_{4}^{U} ; w \wedge^{U}\right)\right\rangle$ and $B=\left\langle\left(b_{1}^{L}, b_{2}^{L}, b_{3}^{L}, b_{4}^{L} ; w \wedge^{L}\right)\right.$ , $\left.\left(b_{1}^{U}, b_{2}^{U}, b_{3}^{U}, b_{4}^{U} ; w \wedge^{U}\right)\right\rangle \in F\left(w \wedge^{L}, w \wedge^{U}\right)$ and $k \in \mathrm{R}$; then,

$A \oplus B=\left\langle\begin{array}{c}\left(a_{1}^{L}+b_{1}^{L}, a_{2}^{L}+b_{2}^{L}, a_{3}^{L}+b_{3}^{L}, a_{4}^{L}+b_{4}^{L} ; w \wedge^{L}\right) \\ \left(a_{1}^{U}+b_{1}^{U}, a_{2}^{U}+b_{2}^{U}, a_{3}^{U}+b_{3}^{U}, a_{4}^{U}+b_{4}^{U} ; w \wedge^{U}\right)\end{array}\right\rangle$,

$k A=\left\{\begin{array}{l}\left\langle\left(k a_{1}^{L}, k a_{2}^{L}, k a_{1}^{L}, k a_{1}^{L} ; w \wedge^{L}\right),\left(k a_{1}^{U}, k a_{2}^{U}, k a_{1}^{U}, k a_{1}^{U} ; w \wedge^{U}\right)\right\rangle, k>0, \\ \left\langle\left(k a_{4}^{L}, k a_{3}^{L}, k a_{2}^{L}, k a_{1}^{L} ; w \wedge^{L}\right),\left(k a_{4}^{U}, k a_{3}^{U}, k a_{2}^{U}, k a_{1}^{U} ; w \wedge^{U}\right)\right\rangle, k<0, \\ \left\langle\left(0,0,0,0 ; w \wedge^{L}\right),\left(0,0,0,0 ; w \wedge^{U}\right)\right\rangle, k=0,\end{array}\right.$

$A \otimes B=\left\langle\begin{array}{c}\left(a_{1}^{L} * b_{1}^{L}, a_{2}^{L} * b_{2}^{L}, a_{3}^{L} * b_{3}^{L}, a_{4}^{L} * b_{4}^{L} ; w \wedge^{L}\right) \\ \left(a_{1}^{U} * b_{1}^{U}, a_{2}^{U} * b_{2}^{U}, a_{3}^{U} * b_{3}^{U}, a_{4}^{U} * b_{4}^{U} ; w \wedge^{U}\right)\end{array}, a_{1}^{U}, b_{1}^{U} \geq 0\right\rangle$.

Definition 7 [28]. Let $\sigma, 0 \in \mathrm{R}$. The signed distance between $\sigma$ and 0 is $d(\sigma, 0)=\sigma$.

Definition 8 [31]. Let $A \in F\left(w \wedge^{L}, w \wedge^{U}\right)$; then, alpha-cut set of A denotes and is defined by

$$
\begin{aligned}
\mathbf{A}(\alpha)= & {\left[A^{L}(\alpha), A^{U}(\alpha)\right] } \\
= & \left\{\left[A_{l}^{U}(\alpha), A_{l}^{L}(\alpha)\right] \cup\left[A_{t}^{L}(\alpha), A_{t}^{U}(\alpha)\right] ;\right. \\
& 0 \leq \alpha \leq w \wedge^{L}\left[A_{l}^{U}(\alpha), A_{t}^{U}(\alpha)\right] ; w \wedge^{L} \leq \alpha \leq w \wedge^{U},
\end{aligned}
$$

where

$$
\begin{aligned}
& A_{l}^{L}(\alpha)=a_{1}^{L}+\left(a_{2}^{L}-a_{1}^{L}\right) \frac{\alpha}{w \wedge^{L}}, \\
& A_{t}^{L}(\alpha)=a_{4}^{L}+\left(a_{4}^{L}-a_{3}^{L}\right) \frac{\alpha}{w \wedge^{L}}, \\
& A_{l}^{U}(\alpha)=a_{1}^{U}+\left(a_{2}^{U}-a_{1}^{U}\right) \frac{\alpha}{w \wedge^{U}}, \\
& A_{t}^{U}(\alpha)=a_{4}^{U}+\left(a_{4}^{U}-a_{3}^{U}\right) \frac{\alpha}{w \wedge^{U}},
\end{aligned}
$$

TABLE 3

\begin{tabular}{lcccc}
\hline$\tau$ & \multicolumn{2}{c}{$\mathrm{MM}$} & \multicolumn{2}{c}{$\mathrm{NN}$} \\
& $\left\|\mathbf{r}_{n+1}-\mathbf{r}_{n}\right\|$ & $\left\|\mathbf{F}\left(\mathbf{r}_{n}\right)\right\|$ & $\left\|\mathbf{r}_{n+1}-\mathbf{r}_{n}\right\|$ & $\left\|\mathbf{F}\left(\mathbf{r}_{n}\right)\right\|$ \\
\hline 0.0 & $4.0 e-49$ & $4.0 e-33$ & $1.7 e-13$ & $1.7 e-11$ \\
0.1 & $5.6 e-47$ & $5.6 e-32$ & $3.4 e-13$ & $1.2 e-11$ \\
0.2 & $3.1 e-43$ & $8.8 e-33$ & $6.1 e-14$ & $3.6 e-11$ \\
0.3 & $5.4 e-48$ & $6.1 e-36$ & $5.1 e-13$ & $7.1 e-11$ \\
0.4 & $6.1 e-49$ & $7.9 e-35$ & $4.4 e-14$ & $5.6 e-12$ \\
0.5 & $3.7 e-48$ & $4.0 e-33$ & $4.3 e-13$ & $3.4 e-11$ \\
0.6 & $1.6 e-48$ & $8.2 e-33$ & $1.7 e-13$ & $4.6 e-11$ \\
0.7 & $3.6 e-49$ & $4.3 e-33$ & $3.7 e-13$ & $6.7 e-11$ \\
0.8 & $1.2 e-49$ & $3.6 e-34$ & $7.1 e-14$ & $5.6 e-11$ \\
0.9 & $7.5 e-46$ & $8.9 e-34$ & $8.7 e-14$ & $1.2 e-11$ \\
1 & $8.1 e-49$ & $6.1 e-35$ & $9.1 e-14$ & $5.1 e-11$ \\
\hline
\end{tabular}

\section{Construction of Iterative Scheme (MM)}

In order to approximate the roots of interval-valued trapezoidal fuzzy nonlinear equation $F(r)=c$, we propose the following two-step iterative scheme as follows:

$$
\left\{\begin{array}{l}
F_{l}^{L}\left(r_{l}^{L}, r_{t}^{L}, r_{l}^{U}, r_{t}^{U}, \tau\right)=c_{l}^{L}(\tau) \\
F_{t}^{L}\left(r_{l}^{L}, r_{t}^{L}, r_{l}^{U}, r_{t}^{U}, \tau\right)=c_{t}^{L}(\tau) \\
F_{l}^{U}\left(r_{l}^{L}, r_{t}^{L}, r_{l}^{U}, r_{t}^{U}, \tau\right)=c_{l}^{U}(\tau) \\
F_{t}^{U}\left(r_{l}^{L}, r_{t}^{L}, r_{l}^{U}, r_{t}^{U}, \tau\right)=c_{t}^{U}(\tau)
\end{array} \forall \tau, 1\right]
$$

Suppose that $r=\left(\alpha_{l}^{L}, \alpha_{t}^{L}, \alpha_{l}^{U}, \alpha_{t}^{U}\right)$ is the solution of above system and $\mathbf{r}_{0}=\left(r_{l 0}^{L}, r_{t 0}^{L}, r_{l 0}^{U}, r_{t 0}^{U}\right)$ is approximate solutions of the system, $t$ denote the alpha-cut parameter; then,

$$
\left\{\begin{array}{l}
\alpha_{l}^{L}(\tau)=r_{l 0}^{L}(\tau)+h_{1}(\tau), \\
\alpha_{l}^{L}(\tau)=r_{t 0}^{L}(\tau)+k_{1}(\tau), \\
\alpha_{l}^{U}(\tau)=r_{l 0}^{U}(\tau)+h_{2}(\tau), \\
\alpha_{t}^{U}(\tau)=r_{t 0}^{U}(\tau)+k_{2}(\tau) .
\end{array}\right.
$$

By using Taylor's series of $F_{l}^{L}, F_{t}^{L}, F_{l}^{U}, F_{t}^{U}$ about $\left(r_{l 0}^{L}(\tau)\right.$, $\left.r_{t 0}^{L}(\tau), r_{l 0}^{U}(\tau), r_{t 0}^{U}(\tau)\right)$, then we have the following: 


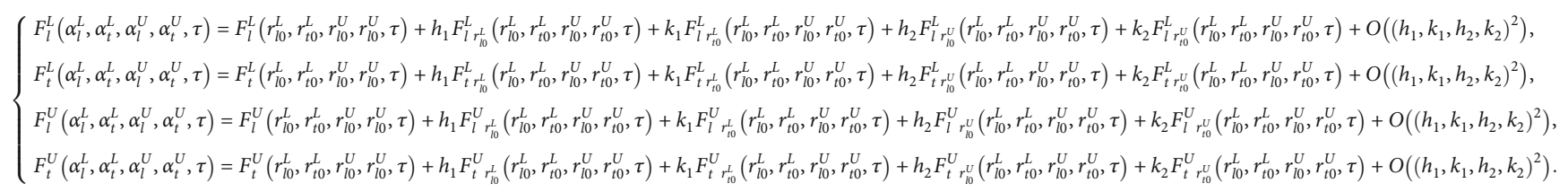

If $\left(r_{l 0}^{L}(\tau), r_{t 0}^{L}(\tau), r_{l 0}^{U}(\tau), r_{t 0}^{U}(\tau)\right)$ are close to $\left(\alpha_{l}^{L}(\tau), \alpha_{l}^{L}(\tau)\right.$ , $\left.\alpha_{l}^{U}(\tau), \alpha_{t}^{U}(\tau)\right)$, then $h_{1}(\tau), k_{1}(\tau), h_{2}(\tau), k_{2}(\tau)$ are small

enough. Assume all partial derivatives of $h_{1}(\tau), k_{1}(\tau), h_{2}(\tau)$ ,$k_{2}(\tau)$ exist and bounded; then, we have the following:

$$
\left\{\begin{array}{l}
F_{l}^{L}\left(r_{l 0}^{L}, r_{t 0}^{L}, r_{l 0}^{U}, r_{l 0}^{U}, \tau\right)+h_{1} F_{l r_{l 0}^{L}}^{L}\left(r_{l 0}^{L}, r_{t 0}^{L}, r_{l 0}^{U}, r_{t 0}^{U}, \tau\right)+k_{1} F_{l r_{t 0}^{L}}^{L}\left(r_{l 0}^{L}, r_{t 0}^{L}, r_{l 0}^{U}, r_{t 0}^{U}, \tau\right)+h_{2} F_{l r_{l 0}^{U}}^{L}\left(r_{l 0}^{L}, r_{t 0}^{L}, r_{l 0}^{U}, r_{t 0}^{U}, \tau\right)+k_{2} F_{l r_{t 0}^{U}}^{L}\left(r_{l 0}^{L}, r_{t 0}^{L}, r_{l 0}^{U}, r_{t 0}^{U}, \tau\right)=c_{l}^{L}(\tau), \\
F_{t}^{L}\left(r_{l 0}^{L}, r_{t 0}^{L}, r_{l 0}^{U}, r_{l 0}^{U}, \tau\right)+h_{1} F_{t r_{l 0}^{L}}^{L}\left(r_{l 0}^{L}, r_{t 0}^{L}, r_{l 0}^{U}, r_{t 0}^{U}, \tau\right)+k_{1} F_{t r_{t 0}^{L}}^{L}\left(r_{l 0}^{L}, r_{t 0}^{L}, r_{l 0}^{U}, r_{t 0}^{U}, \tau\right)+h_{2} F_{t r_{l 0}^{U}}^{L}\left(r_{l 0}^{L}, r_{t 0}^{L}, r_{l 0}^{U}, r_{t 0}^{U}, \tau\right)+k_{2} F_{t r_{t 0}^{U}}^{L}\left(r_{l 0}^{L}, r_{t 0}^{L}, r_{l 0}^{U}, r_{t 0}^{U}, \tau\right)=c_{t}^{L}(\tau), \\
F_{l}^{U}\left(r_{l 0}^{L}, r_{t 0}^{L}, r_{l 0}^{U}, r_{l 0}^{U}, \tau\right)+h_{1} F_{l r_{l 0}^{L}}^{U}\left(r_{l 0}^{L}, r_{t 0}^{L}, r_{l 0}^{U}, r_{t 0}^{U}, \tau\right)+k_{1} F_{l r_{t 0}^{L}}^{U}\left(r_{l 0}^{L}, r_{t 0}^{L}, r_{l 0}^{U}, r_{t 0}^{U}, \tau\right)+h_{2} F_{l r_{l 0}^{U}}^{U}\left(r_{l 0}^{L}, r_{t 0}^{L}, r_{l 0}^{U}, r_{t 0}^{U}, \tau\right)+k_{2} F_{l}^{U} r_{t 0}^{U}\left(r_{l 0}^{L}, r_{t 0}^{L}, r_{l 0}^{U}, r_{t 0}^{U}, \tau\right)=c_{l}^{L}(\tau) \\
F_{t}^{U}\left(r_{l 0}^{L}, r_{t 0}^{L}, r_{l 0}^{U}, r_{l 0}^{U}, \tau\right)+h_{1} F_{t r_{l 0}^{L}}^{U}\left(r_{l 0}^{L}, r_{t 0}^{L}, r_{l 0}^{U}, r_{t 0}^{U}, \tau\right)+k_{1} F_{t r_{t 0}^{L}}^{U}\left(r_{l 0}^{L}, r_{t 0}^{L}, r_{l 0}^{U}, r_{t 0}^{U}, \tau\right)+h_{2} F_{t r_{l 0}^{U}}^{U}\left(r_{l 0}^{L}, r_{t 0}^{L}, r_{l 0}^{U}, r_{t 0}^{U}, \tau\right)+k_{2} F_{t r_{t 0}^{U}}^{U}\left(r_{l 0}^{L}, r_{t 0}^{L}, r_{l 0}^{U}, r_{t 0}^{U}, \tau\right)=c_{t}^{L}(\tau)
\end{array}\right.
$$

Since $h_{1}(\tau), k_{1}(\tau), h_{2}(\tau), k_{2}(\tau)$ are unknown quantities, they are obtained by solving the following equations:

$J_{*}\left(r_{l 0}^{L}, r_{t 0}^{L}, r_{l 0}^{U}, r_{t 0}^{U}, \tau\right)\left[\begin{array}{l}h_{1}(\tau) \\ k_{1}(\tau) \\ h_{2}(\tau) \\ k_{2}(\tau)\end{array}\right]=\left[\begin{array}{c}c_{l}^{L}(\tau)-F_{l}^{L}\left(r_{l 0}^{L}, r_{t 0}^{L}, r_{l 0}^{U}, r_{t 0}^{U}, \tau\right) \\ c_{t}^{L}(\tau)-F_{t}^{L}\left(r_{l 0}^{L}, r_{t 0}^{L}, r_{l 0}^{U}, r_{t 0}^{U}, \tau\right) \\ c_{l}^{U}(\tau)-F_{l}^{U}\left(r_{l 0}^{L}, r_{t 0}^{L}, r_{l 0}^{U}, r_{t 0}^{U}, \tau\right) \\ c_{t}^{U}(\tau)-F_{t}^{U}\left(r_{l 0}^{L}, r_{t 0}^{L}, r_{l 0}^{U}, r_{t 0}^{U}, \tau\right)\end{array}\right]$,

where

$$
\mathbf{J}_{*}=\left[\begin{array}{cccc}
F_{l r_{l 0}^{L}}^{L}\left(r_{l 0}^{L}, r_{t 0}^{L}, r_{l 0}^{U}, r_{t 0}^{U}, \tau\right) & F_{l r_{t 0}^{L}}^{L}\left(r_{l 0}^{L}, r_{t 0}^{L}, r_{l 0}^{U}, r_{t 0}^{U}, \tau\right) & F_{l r_{l 0}^{U}}^{L}\left(r_{l 0}^{L}, r_{t 0}^{L}, r_{l 0}^{U}, r_{t 0}^{U}, \tau\right) & F_{l r_{t 0}^{U}}^{L}\left(r_{l 0}^{L}, r_{t 0}^{L}, r_{l 0}^{U}, r_{t 0}^{U}, \tau\right) \\
F_{t r_{l 0}^{L}}^{L}\left(r_{l 0}^{L}, r_{t 0}^{L}, r_{l 0}^{U}, r_{t 0}^{U}, \tau\right) & F_{t r_{t 0}^{L}}^{L}\left(r_{l 0}^{L}, r_{t 0}^{L}, r_{l 0}^{U}, r_{t 0}^{U}, \tau\right) & F_{t r_{l 0}^{U}}^{L}\left(r_{l 0}^{L}, r_{t 0}^{L}, r_{l 0}^{U}, r_{t 0}^{U}, \tau\right) & F_{t r_{t 0}^{U}}^{L}\left(r_{l 0}^{L}, r_{t 0}^{L}, r_{l 0}^{U}, r_{t 0}^{U}, \tau\right) \\
F_{l r_{l 0}^{L}}^{U}\left(r_{l 0}^{L}, r_{t 0}^{L}, r_{l 0}^{U}, r_{t 0}^{U}, \tau\right) & F_{l r_{t 0}^{L}}^{U}\left(r_{l 0}^{L}, r_{t 0}^{L}, r_{l 0}^{U}, r_{t 0}^{U}, \tau\right) & F_{l r_{l 0}^{U}}^{U}\left(r_{l 0}^{L}, r_{t 0}^{L}, r_{l 0}^{U}, r_{t 0}^{U}, \tau\right) & F_{l r_{t 0}^{U}}^{U}\left(r_{l 0}^{L}, r_{t 0}^{L}, r_{l 0}^{U}, r_{t 0}^{U}, \tau\right) \\
F_{t r_{l 0}^{L}}^{U}\left(r_{l 0}^{L}, r_{t 0}^{L}, r_{l 0}^{U}, r_{t 0}^{U}, \tau\right) & F_{t r_{t 0}^{L}}^{U}\left(r_{l 0}^{L}, r_{t 0}^{L}, r_{l 0}^{U}, r_{t 0}^{U}, \tau\right) & F_{t r_{l 0}^{U}}^{U}\left(r_{l 0}^{L}, r_{t 0}^{L}, r_{l 0}^{U}, r_{t 0}^{U}, \tau\right) & F_{t r_{t 0}^{U}}^{U}\left(r_{l 0}^{L}, r_{t 0}^{L}, r_{l 0}^{U}, r_{t 0}^{U}, \tau\right)
\end{array}\right] .
$$

$$
\left[\begin{array}{c}
y_{l 0}^{L}(\tau) \\
y_{t 0}^{L}(\tau) \\
y_{l 0}^{U}(\tau) \\
y_{t 0}^{U}(\tau)
\end{array}\right]=\left[\begin{array}{c}
r_{l 0}^{L}(\tau) \\
r_{t 0}^{L}(\tau) \\
r_{l 0}^{U}(\tau) \\
r_{t 0}^{U}(\tau)
\end{array}\right]+\left[\begin{array}{l}
h_{1}(\tau) \\
k_{1}(\tau) \\
h_{2}(\tau) \\
k_{2}(\tau)
\end{array}\right]
$$




$$
\begin{gathered}
{\left[\begin{array}{l}
r_{l 1}^{L}(\tau) \\
r_{t 1}^{L}(\tau) \\
r_{l 1}^{U}(\tau) \\
r_{t 1}^{U}(\tau)
\end{array}\right]=\left[\begin{array}{l}
y_{l 0}^{L}(\tau) \\
y_{t 0}^{L}(\tau) \\
y_{l 0}^{U}(\tau) \\
y_{t 0}^{U}(\tau)
\end{array}\right]+Z *\left[\begin{array}{l}
h_{1}(\tau) \\
k_{1}(\tau) \\
h_{2}(\tau) \\
k_{2}(\tau)
\end{array}\right],} \\
\mathbf{J}_{* *}=\left[\begin{array}{llll}
F_{l 0} \\
F_{l y_{l 0}^{L}}^{L}\left(y_{l 0}^{L}, y_{t 0}^{L}, y_{l 0}^{U}, y_{t 0}^{U}, \tau\right) & F_{l y_{t 0}^{L}}^{L}\left(y_{l 0}^{L}, y_{t 0}^{L}, y_{l 0}^{U}, y_{t 0}^{U}, \tau\right) & F_{l y_{l 0}^{U}}^{L}\left(y_{l 0}^{L}, y_{t 0}^{L}, y_{l 0}^{U}, y_{t 0}^{U}, \tau\right) & F_{l y_{t 0}^{U}}^{L}\left(y_{l 0}^{L}, y_{t 0}^{L}, y_{l 0}^{U}, y_{t 0}^{U}, \tau\right) \\
F_{t y_{l 0}^{L}}^{L}\left(y_{l 0}^{L}, y_{t 0}^{L}, y_{l 0}^{U}, y_{t 0}^{U}, \tau\right) & F_{t y_{t 0}^{L}}^{L}\left(y_{l 0}^{L}, y_{t 0}^{L}, y_{l 0}^{U}, y_{t 0}^{U}, \tau\right) & F_{t y_{l 0}^{U}}^{L}\left(y_{l 0}^{L}, y_{t 0}^{L}, y_{l 0}^{U}, y_{t 0}^{U}, \tau\right) & F_{t y_{t 0}^{U}}^{L}\left(y_{l 0}^{L}, y_{t 0}^{L}, y_{l 0}^{U}, y_{t 0}^{U}, \tau\right) \\
F_{l y_{l 0}^{L}}^{U}\left(y_{l 0}^{L}, y_{t 0}^{L}, y_{l 0}^{U}, y_{t 0}^{U}, \tau\right) & F_{l y_{t 0}^{L}}^{U}\left(y_{l 0}^{L}, y_{t 0}^{L}, y_{l 0}^{U}, y_{t 0}^{U}, \tau\right) & F_{l y_{10}^{U}}^{U}\left(y_{l 0}^{L}, y_{t 0}^{L}, y_{l 0}^{U}, y_{t 0}^{U}, \tau\right) & F_{l y_{t 0}^{U}}^{U}\left(y_{l 0}^{L}, y_{t 0}^{L}, y_{l 0}^{U}, y_{t 0}^{U}, \tau\right) \\
F_{t y_{l 0}^{L}}^{U}\left(y_{l 0}^{L}, y_{t 0}^{L}, y_{l 0}^{U}, y_{t 0}^{U}, \tau\right) & F_{t y_{t 0}^{L}}^{U}\left(y_{l 0}^{L}, y_{t 0}^{L}, y_{l 0}^{U}, y_{t 0}^{U}, \tau\right) & F_{t y_{l 0}^{U}}^{U}\left(y_{l 0}^{L}, y_{t 0}^{L}, y_{l 0}^{U}, y_{t 0}^{U}, \tau\right) & F_{t y_{t 0}^{U}}^{U}\left(y_{l 0}^{L}, y_{t 0}^{L}, y_{l 0}^{U}, y_{t 0}^{U}, \tau\right)
\end{array}\right] .
\end{gathered}
$$

$\mathbf{J}_{*}=J_{*}\left(r_{\ln }^{L}, r_{t n}^{L}, r_{\ln }^{U}, r_{t n}^{U}, \tau\right), \quad \mathbf{J}_{* *}=J_{* *}\left(y_{\ln }^{L}, y_{t n}^{L}, y_{\ln }^{U}, y_{t n}^{U}, \tau\right)$, and the next approximation for $r_{l}^{L}(\tau), r_{t}^{L}(\tau), r_{l}^{U}(\tau), r_{t}^{U}(\tau)$ is found by using recursive scheme as follows:

$$
\begin{aligned}
& {\left[\begin{array}{c}
r_{l_{(n+1)}^{L}}^{L}(\tau) \\
r_{t_{(n+1)}^{L}}^{L}(\tau) \\
r_{l_{(n+1)}^{U}(\tau)}^{U} \\
r_{t(n+1)}^{U}(\tau)
\end{array}\right]=\left[\begin{array}{l}
y_{l(n)}^{L}(\tau) \\
y_{t(n)}^{L}(\tau) \\
y_{l_{(n)}}^{U}(\tau) \\
y_{t(n)}^{U}(\tau)
\end{array}\right]+Z *\left[\begin{array}{l}
h_{1 n}(\tau) \\
k_{1 n}(\tau) \\
h_{2 n}(\tau) \\
k_{2 n}(\tau)
\end{array}\right]} \\
& \begin{array}{l}
y_{l(n)}^{L}(\tau) \\
y_{t(n)}^{L}(\tau) \\
y_{l(n)}^{U}(\tau) \\
y_{t(n)}^{U}(\tau)
\end{array}=\left[\begin{array}{c}
r_{l(n)}^{L}(\tau) \\
r_{t(n)}^{L}(\tau) \\
r_{l(n)}^{U}(\tau) \\
r_{t(n)}^{U}(\tau)
\end{array}\right]+\left[\begin{array}{l}
h_{1 n}(\tau) \\
k_{1 n}(\tau) \\
h_{2 n}(\tau) \\
k_{2 n}(\tau)
\end{array}\right] \\
& Z=\left(4 J_{* *}\left(y_{l(n)}^{L}, y_{t(n)}^{L}, y_{l(n)}^{U}, y_{t(n)}^{U}, \tau\right)-2 J_{*}\left(r_{l(n)}^{L}, r_{t(n)}^{L}, r_{l(n)}^{U}, r_{t(n)}^{U}, \tau\right)\right)^{-1} *\left(J_{*}\left(y_{l(n)}^{L}, y_{t(n)}^{L}, y_{l(n)}^{U}, y_{t(n)}^{U}, \tau\right)-J_{* *}\left(r_{l(n)}^{L}, r_{t(n)}^{L}, r_{l(n)}^{U}, r_{t(n)}^{U}, \tau\right)\right. \\
& J_{*}\left(r_{\mathrm{ln}}^{L}, r_{t n}^{L}, r_{\mathrm{ln}}^{U}, r_{t n}^{U}, \tau\right)\left[\begin{array}{l}
h_{1 n}(\tau) \\
k_{1 n}(\tau) \\
h_{2 n}(\tau) \\
k_{2 n}(\tau)
\end{array}\right]=\left[\begin{array}{c}
c_{l}^{L}(\tau)-F_{l}^{L}\left(r_{\mathrm{ln}}^{L}, r_{t n}^{L}, r_{\mathrm{ln}}^{U}, r_{t n}^{U}, \tau\right) \\
c_{t}^{L}(\tau)-F_{t}^{L}\left(r_{\mathrm{ln}}^{L}, r_{t n}^{L}, r_{\mathrm{ln}}^{U}, r_{t n}^{U}, \tau\right) \\
c_{l}^{U}(\tau)-F_{l}^{U}\left(r_{\mathrm{ln}}^{L}, r_{t n}^{L}, r_{\mathrm{ln}}^{U}, r_{t n}^{U}, \tau\right) \\
c_{t}^{U}(\tau)-F_{t}^{U}\left(r_{\mathrm{ln}}^{L}, r_{t n}^{L}, r_{\mathrm{ln}}^{U}, r_{t n}^{U}, \tau\right)
\end{array}\right],
\end{aligned}
$$




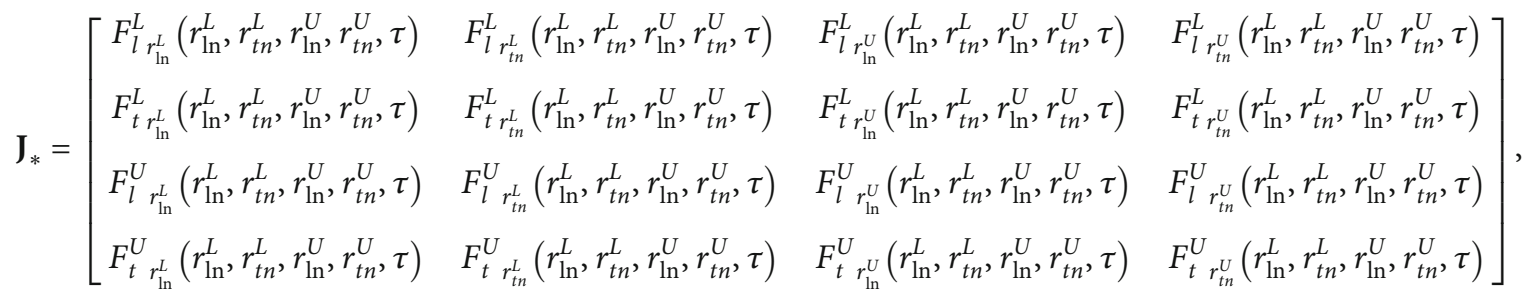

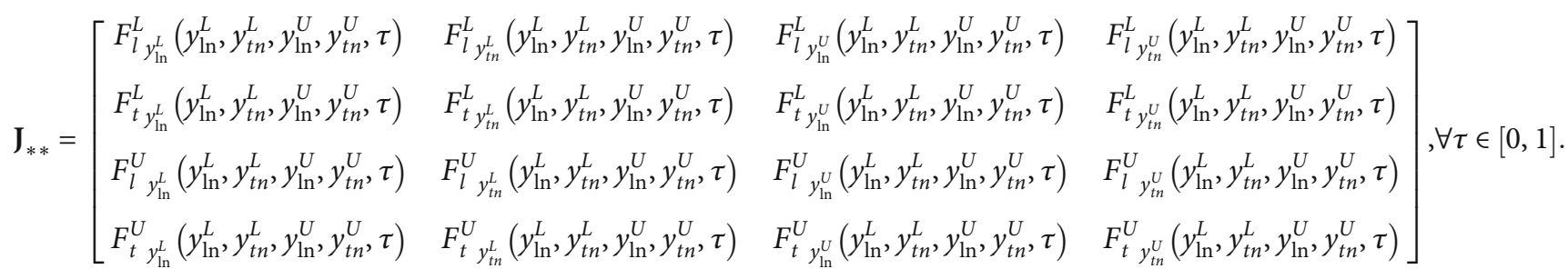

For initial guess, one can use the fuzzy number

$\left.\mathbf{r}_{0}=\left(r_{l 0}^{U}(0), r_{l 0}^{L}(0)\right), r_{l 0}^{L}(1), r_{t 0}^{L}(1), r_{l 0}^{U}(1), r_{t 0}^{U}(1), r_{t 0}^{L}(0) r_{t 0}^{U}(0)\right)$,

Remark 9. Sequence $\left\{\left(r_{\ln }^{L}, r_{t n}^{L}, r_{\ln }^{U}, r_{t n}^{U}\right)\right\}_{n=0}^{\infty}$ converges to $\left(\alpha_{l}^{L}\right.$, $\left.\alpha_{t}^{L}, \alpha_{l}^{U}, \alpha_{t}^{U}\right)$ iff $\forall \tau \in[0,1], \lim _{n \longrightarrow \infty} r_{\ln }^{L}(\tau)=\alpha_{l}^{L}(\tau), \lim _{n \longrightarrow \infty}$ $r_{t n}^{L}(\tau)=\alpha_{t}^{L}(\tau), \lim _{n \longrightarrow \infty} r_{\ln }^{U}(\tau)=\alpha_{l}^{U}(\tau)$, and $\lim _{n \longrightarrow \infty} r_{t n}^{U}(\tau)$ $=\alpha_{t}^{U}(\tau)$.

Lemma 10. Let $F\left(\alpha_{l}^{L}, \alpha_{t}^{L}, \alpha_{l}^{U}, \alpha_{t}^{U}\right)=\left(c_{l}^{L}, c_{t}^{L}, c_{l}^{U}, c_{t}^{U}\right)$, and if the sequence of $\left\{\left(r_{\ln }^{L}, r_{t n}^{L}, r_{\ln }^{U}, r_{t n}^{U}\right)\right\}_{n=0}^{\infty}$ converges to $\left(\alpha_{l}^{L}, \alpha_{t}^{L}, \alpha_{l}^{U}\right.$, $\alpha_{t}^{U}$ ) according to Newton's method, then

$$
\lim _{n \longrightarrow \infty} \mathbf{P}_{n}=0
$$

where

$$
\mathbf{P}_{n}=\sup _{0 \leq \tau \leq 1} \max \left\{h_{1 n}(\tau), k_{1 n}(\tau), h_{2 n}(\tau), k_{2 n}(\tau)\right\}
$$

Proof. It is obvious, because for all $\forall \tau \in[0,1]$ in convergent case,

$\lim _{n \longrightarrow \infty} h_{1 n}(\tau)=\lim _{n \longrightarrow \infty} k_{1 n}(\tau)=\lim _{n \longrightarrow \infty} h_{2 n}(\tau)=\lim _{n \longrightarrow \infty} k_{2 n}(\tau)=0$.

Hence, it is proved.

Finally, it is shown that under certain condition, the MM method for fuzzy equation $\mathbf{F}(\mathbf{r})=0$ is cubic convergent. In compact form for $\mathbf{F}(\mathbf{r})=0$, the MM method can be written as follows:

$$
\left\{\begin{array}{l}
\mathbf{y}_{n}(\tau)=\mathbf{r}_{n}(\tau)-\left(\mathbf{F}^{\prime}\left(\mathbf{r}_{n}(\tau)\right)\right)^{-1} \mathbf{F}\left(\mathbf{r}_{n}(\tau)\right), \\
\mathbf{r}_{n+1}(\tau)=\mathbf{y}_{n}(\tau)-Z *\left(\mathbf{F}^{\prime}\left(\mathbf{r}_{n}(\tau)\right)\right)^{-1} \mathbf{F}\left(\mathbf{r}_{n}(\tau)\right),
\end{array} \forall \tau \in[0,1],\right.
$$

where

$$
Z=\left(4 \mathbf{J}_{* *}-2 \mathbf{J}_{*}\right)^{-1} *\left(\mathbf{J}_{*}-\mathbf{J}_{* *}\right) .
$$

Theorem 11. Let $F: H \subseteq R^{n} \longrightarrow R^{n}$, be u-times Frĕchet differential function on a convex set $H$ containing the root $\boldsymbol{\alpha}$ of $\mathbf{F}(\mathbf{r})=0$; then, the MM method has cubic convergence and satisfies the following error equation.

$$
\mathbf{e}_{n+1}=2 *\left(\left(\mathbf{A}_{2}\right)^{2}-\frac{1}{2} \mathbf{A}_{3}-4\left(\mathbf{A}_{2}\right)^{2}\right)\left(\mathbf{e}_{n}\right)^{3}+\left\|\mathbf{O}\left(\mathbf{e}_{n}\right)^{4}\right\|,
$$

where $\mathbf{A}_{n}=1 / 2 ! * \mathbf{F}\left(\mathbf{r}_{n}, \tau\right) / \mathbf{F}^{\prime}\left(\mathbf{r}_{n}, \tau\right), n=2,3, \cdots$.

Proof. Let $\mathbf{e}_{n}=\mathbf{r}_{n}-\alpha$ and $\mathbf{e}_{n+1}=\mathbf{r}_{n+1}-\boldsymbol{\alpha}$, then by Taylor series of $\mathbf{F}\left(\mathbf{r}_{n}, \tau\right)$ in the neighborhood of $\boldsymbol{\alpha}$ if $\mathbf{J}_{*}$ and $\mathbf{J}_{* *}$ exist. Then,

$\mathbf{F}(\mathbf{r}, \tau)=\mathbf{F}\left(\mathbf{r}_{n}, \tau\right)+\mathbf{F}^{\prime}\left(\mathbf{r}_{n}, \tau\right)\left(\mathbf{r}-\mathbf{r}_{n}\right)+\frac{1}{2 !} \mathbf{F}^{\prime \prime}\left(\mathbf{r}_{n}, \tau\right)\left(\mathbf{r}-\mathbf{r}_{n}\right)^{2}+\ldots$

$$
\text { and } \mathbf{F}(\mathbf{r}, \boldsymbol{\alpha})=0 \text {. }
$$

$$
\mathbf{F}\left(\mathbf{r}_{n}, \tau\right)=\mathbf{F}^{\prime}(\mathbf{r}, \xi)\left(\mathbf{e}_{n}+\mathbf{A}_{2}\left(\mathbf{e}_{n}\right)^{2}+\mathbf{A}_{3}\left(\mathbf{e}_{n}\right)^{3}\right)+\left\|\mathbf{O}\left(\mathbf{e}_{n}\right)^{4}\right\| .
$$


TABle 4: Analytical solution for Example 2.

\begin{tabular}{ccccccccccccc}
\hline$\tau$ & 0.0 & 0.1 & 0.2 & 0.3 & 0.4 & 0.5 & 0.6 & 0.7 & 0.8 & 0.9 & 1.0 \\
$r_{l}^{U}$ & 0.6433 & 0.6437 & 0.6440 & 0.6443 & 0.6446 & 0.6450 & 0.6453 & 0.6457 & 0.6460 & 0.6464 & 0.6468 \\
$r_{l}^{L}$ & 0.6450 & 0.6454 & 0.6458 & 0.6463 & 0.6467 & 0.6472 & 0.6477 & 0.6482 & 0.6487 & 0.6493 & 0.6498 \\
$r_{t}^{L}$ & 0.6616 & 0.6606 & 0.6596 & 0.6586 & 0.6577 & 0.6568 & 0.6560 & 0.6552 & 0.6544 & 0.6537 & 0.6530 \\
$r_{t}^{U}$ & 0.6666 & 0.6655 & 0.6645 & 0.6635 & 0.6625 & 0.6616 & 0.6607 & 0.6599 & 0.6591 & 0.6583 & 0.6576 \\
\hline
\end{tabular}

This gives

$$
\begin{gathered}
\left(\mathbf{F}^{\prime}\left(\mathbf{r}_{n}, \tau\right)\right)^{-1} \mathbf{F}\left(\mathbf{r}_{n}, \tau\right)=\mathbf{e}_{n}+\mathbf{A}_{2}\left(\mathbf{e}_{n}\right)^{2}+\left(2 \mathbf{A}_{2}+2 \mathbf{A}_{3}\right)\left(\mathbf{e}_{n}\right)^{3}+\cdots \\
\mathbf{y}_{n}-\boldsymbol{\alpha}=\mathbf{A}_{2}\left(\mathbf{e}_{n}\right)^{2}+\left(-2 \mathbf{A}_{2}+2 \mathbf{A}_{3}\right)\left(e_{n}\right)^{3}+. \cdots
\end{gathered}
$$

Expanding $\mathbf{F}^{\prime}\left(\mathbf{y}_{n}, \tau\right)$ about $\boldsymbol{\alpha}$, we have the following:

$$
\begin{aligned}
& \mathbf{F}^{\prime}\left(\mathbf{y}_{n}, \tau\right)=1+2\left(\mathbf{A}_{2}\right)^{2}\left(\mathbf{e}_{n}\right)^{2}+2\left(-2\left(\mathbf{A}_{2}\right)^{2}+2 \mathbf{A}_{3}\right)\left(\mathbf{e}_{n}\right)^{3}+\cdots \\
& Z *\left(\mathbf{F}^{\prime}\left(\mathbf{r}_{n}, \tau\right)\right)^{-1} \mathbf{F}\left(\mathbf{r}_{n}, \tau\right)=-\mathbf{A}_{2}\left(\mathbf{e}_{n}\right)^{2}+\left(4\left(\mathbf{A}_{2}\right)^{2}-\frac{3}{2} \mathbf{A}_{3}-4\left(\mathbf{A}_{2}\right)^{2}\right)\left(\mathbf{e}_{n}\right)^{3}+\cdots,
\end{aligned}
$$

$$
\begin{aligned}
& \mathbf{r}_{n+1}-\boldsymbol{\alpha}=\mathbf{y}_{n}-\boldsymbol{\alpha}-\mathbf{A}_{2}\left(\mathbf{e}_{n}\right)^{2}+\left(4\left(\mathbf{A}_{2}\right)^{2}-\frac{3}{2} \mathbf{A}_{3}-4\left(\mathbf{A}_{2}\right)^{2}\right)\left(\mathbf{e}_{n}\right)^{3}+\cdots \\
& \mathbf{e}_{n+1}=2 *\left(\left(\mathbf{A}_{2}\right)^{2}-\frac{1}{2} \mathbf{A}_{3}-4\left(\mathbf{A}_{2}\right)^{2}\right)\left(\mathbf{e}_{n}\right)^{3}+\left\|\mathbf{O}\left(e_{n}\right)^{4}\right\| .
\end{aligned}
$$

Hence, the theorem is proved.

A well-known existing method in literature for solving triangular fuzzy nonlinear equation is classical Newton Raphson's method. Interval-valued trapezoidal fuzzy version of well-known Newton method [13] (abbreviated as NN) for finding roots of interval-valued trapezoidal fuzzy nonlinear equation is as follows:

$$
\left[\begin{array}{c}
r_{l(n+1)}^{L}(\tau) \\
r_{t(n+1)}^{L}(\tau) \\
r_{l_{(n+1)}^{U}(\tau)}^{U} \\
r_{t(n+1)}^{U}(\tau)
\end{array}\right]=\left[\begin{array}{c}
r_{l(n)}^{L}(\tau) \\
r_{t(n)}^{L}(\tau) \\
r_{l(n)}^{U}(\tau) \\
r_{t(n)}^{U}(\tau)
\end{array}\right]+\left[\begin{array}{l}
h_{1 n}(\tau) \\
k_{1 n}(\tau) \\
h_{2 n}(\tau) \\
k_{2 n}(\tau)
\end{array}\right]
$$

TABLE 5

\begin{tabular}{lcccc}
\hline$\tau$ & \multicolumn{2}{c}{$\mathrm{MM}$} & \multicolumn{2}{c}{$\mathrm{NN}$} \\
& $\left\|\mathbf{r}_{n+1}-\mathbf{r}_{n}\right\|$ & $\left\|\mathbf{F}\left(\mathbf{r}_{n}\right)\right\|$ & $\left\|\mathbf{r}_{n+1}-\mathbf{r}_{n}\right\|$ & $\left\|\mathbf{F}\left(\mathbf{r}_{n}\right)\right\|$ \\
\hline 0.0 & $2.8 e-43$ & $5.4 e-33$ & $1.1 e-9$ & $1.6 e-7$ \\
0.1 & $3.8 e-44$ & $3.0 e-33$ & $7.6 e-10$ & $3.4 e-7$ \\
0.2 & $4.7 e-43$ & $5.8 e-33$ & $3.5 e-10$ & $4.2 e-7$ \\
0.3 & $9.1 e-43$ & $2.7 e-33$ & $6.1 e-11$ & $6.1 e-7$ \\
0.4 & $6.2 e-50$ & $5.6 e-39$ & $5.1 e-12$ & $5.8 e-7$ \\
0.5 & $3.6 e-51$ & $6.1 e-38$ & $4.6 e-11$ & $6.5 e-7$ \\
0.6 & $4.8 e-50$ & $7.8 e-39$ & $3.1 e-11$ & $8.9 e-7$ \\
0.7 & $9.1 e-51$ & $3.6 e-39$ & $4.2 e-11$ & $1.3 e-7$ \\
0.8 & $8.1 e-48$ & $8.4 e-40$ & $7.7 e-11$ & $4.1 e-7$ \\
0.9 & $9.8 e-49$ & $3.5 e-39$ & $5.1 e-11$ & $5.6 e-7$ \\
1 & $6.1 e-52$ & $4.0 e-38$ & $3.3 e-11$ & $1.2 e-7$ \\
\hline
\end{tabular}

where

$$
J_{*}\left(r_{\ln }^{L}, r_{t n}^{L}, r_{\ln }^{U}, r_{t n}^{U}, \tau\right)\left[\begin{array}{c}
h_{1 n}(\tau) \\
k_{1 n}(\tau) \\
h_{2 n}(\tau) \\
k_{2 n}(\tau)
\end{array}\right]=\left[\begin{array}{c}
c_{l}^{L}(\tau)-F_{l}^{L}\left(r_{\ln }^{L}, r_{t n}^{L}, r_{\ln }^{U}, r_{t n}^{U}, \tau\right) \\
c_{t}^{L}(\tau)-F_{t}^{L}\left(r_{\ln }^{L}, r_{t n}^{L}, r_{\ln }^{U}, r_{t n}^{U}, \tau\right) \\
c_{l}^{U}(\tau)-F_{l}^{U}\left(r_{\ln }^{L}, r_{t n}^{L}, r_{\ln }^{U}, r_{t n}^{U}, \tau\right) \\
c_{t}^{U}(\tau)-F_{t}^{U}\left(r_{\ln }^{L}, r_{t n}^{L}, r_{\ln }^{U}, r_{t n}^{U}, \tau\right)
\end{array}\right] .
$$

\section{Numerical Applications}

Here, we present examples to illustrate the performance and efficiency of MM and NN methods for approximating roots of interval-valued trapezoidal fuzzy nonlinear equations. Examples 1-3 are considered from Buckley and Qu [9]. All the computations are performed using CAS Maple 18 with 64 digits floating point arithmetic with stopping criteria as follows. Analytical, numerical approximate solutions, computational order of convergence [32], computational time in second, and residual error graph of interval-valued trapezoidal fuzzy nonlinear equation used in Examples 1-3 are shown in Figures 2-8(a) and 8(c), respectively. Algorithm 1 shows the implementation of MM iterative method on CAS Maple18.

$$
\text { (i) } e_{n}=\|F(r, \tau)\|<\epsilon \quad(\text { ii }) e_{n}=\left\|r_{n+1}(\tau)-r_{n}(\tau)\right\|<\epsilon,
$$

where $e_{n}$ represents the absolute error. We take $\epsilon=10^{-15}$.

In Figure 2, left shows analytical solution of intervalvalued trapezoidal fuzzy nonlinear equation used in Example 
TABle 6: Analytical solution for Example 3.

\begin{tabular}{|c|c|c|c|c|c|c|c|c|c|c|c|}
\hline$\tau$ & 0.0 & 0.1 & 0.2 & 0.3 & 0.4 & 0.5 & 0.6 & 0.7 & 0.8 & 0.9 & 1.0 \\
\hline$r_{l}^{U}$ & 0.3173 & 0.3266 & 0.3355 & 0.3440 & 0.3522 & 0.3600 & 0.3675 & 0.3747 & 0.3817 & 0.3883 & 0.3947 \\
\hline$r_{l}^{L}$ & 0.3600 & 0.3690 & 0.3775 & 0.3857 & 0.3935 & 0.4009 & 0.4080 & 0.4148 & 0.4213 & 0.4276 & 0.4336 \\
\hline$r_{t}^{L}$ & 0.5005 & 0.4971 & 0.4935 & 0.4898 & 0.4860 & 0.4821 & 0.4780 & 0.4738 & 0.4694 & 0.4648 & 0.4601 \\
\hline$r_{t}^{U}$ & 0.5137 & 0.5112 & 0.5087 & 0.5060 & 0.5033 & 0.5005 & 0.4977 & 0.4947 & 0.4917 & 0.4886 & 0.4854 \\
\hline
\end{tabular}

1, center shows for Example 2, and right shows for Example 3 , respectively.

Figure 3 shows computational order of convergence of iterative methods $\mathrm{MM}$ and $\mathrm{NN}$ for finding roots of intervalvalued trapezoidal fuzzy nonlinear equations used in Examples $1-3$, respectively.

In Figure 3, MM1-MM4 and NN1-NN4 show computational order of convergence of iterative method MM and $\mathrm{NN}$ for approximating roots of interval-valued trapezoidal fuzzy nonlinear equations used in Examples 1-3, respectively.

Figure 4 shows computational time in seconds of iterative methods MM and NN for finding roots of interval-valued trapezoidal fuzzy nonlinear equations used in Examples 13 , respectively.

In Figure 4, MM1-MM4 and NN1-NN4 show computational time in seconds of iterative method $\mathrm{MM}$ and $\mathrm{NN}$ for finding roots of interval-valued trapezoidal fuzzy nonlinear equation used in Examples 1-3, respectively.

Example 1 Application in optimization (a profit maximization problem). A corporation company wishes to invest one million dollar $A_{1}=\langle(10,20,30,40 ; 2 / 3),(5,15,35,43 ; 1)\rangle$ at fuzzy interest rate $r$ to earn maximum profit, so that after a year, they may withdraw $25000 \$ S_{1}=\langle(45,55,75,95 ; 2 / 3)$ , $(80,90,110,120 ; 1)\rangle$ approximately and after two years $900000 \$ S_{2}=\langle(10,15,20,25 ; 2 / 3),(5,10,25,47 ; 1)\rangle \quad$ left. Find $r$ so that $A_{1}$ will be sufficient to cover $S_{1}$ and $S_{2}$. where $r$ is an interval-valued trapezoidal fuzzy number whose support lies between $[0,1]$ After a year, the amount in the account will be

$$
\left(A_{1}-S_{1}\right)+A_{1} r(\tau) .
$$

At the end of second year, total amount left is

$$
\left(A_{1}-S_{1}\right)+A_{1} * r(\tau)+\left(\left(A_{1}-S_{1}\right)-A_{1} * r(\tau)\right) r(\tau),
$$

or

$$
A_{1}\left(r(\tau)^{2}\right)+B * r(\tau)+D,
$$

where $B=2 A_{1}-S_{1}$ and $D=A_{1}-S_{1}$. Therefore, we have to solve

$$
A_{1} *(r(\tau))^{2}+B * r(\tau)+D=S_{2},
$$

or

$$
A_{1} *\left(r(\tau)^{2}\right)+B * r(\tau)=C,
$$

where $C=S_{2}-D$. For fuzzy interest rate substituting values of $A_{1}, B$, and $C$ in above equation, we have the following:

$$
\begin{aligned}
& \left\langle\left(10,20,30,40 ; \frac{2}{3}\right),(5,15,35,45 ; 1)\right\rangle\left(r(\tau)^{2}\right) \\
& \quad+\left\langle\left(50,60,70,80 ; \frac{2}{3}\right),(45,55,75,95 ; 1)\right\rangle r(\tau) \\
& =\left\langle\left(80,90,110,120 ; \frac{2}{3}\right),(75,85,115,125 ; 1)\right\rangle .
\end{aligned}
$$

Without any loss of generality, assume that $r$ is positive; then, the parametric form of this equation is as follows:

$$
\begin{aligned}
&\left\{\langle(10+15 \tau, 40-15 \tau),(5+10 \tau, 45-10 \tau)\rangle(r(\tau))^{2}\right. \\
&+\langle(50+15 \tau, 80-15 \tau),(45+10 \tau, 95-20 \tau)\rangle r(\tau) \\
&=\langle(80+15 \tau, 120-15 \tau),(75+10 \tau, 125-10 \tau)\rangle,
\end{aligned}
$$

$$
\left\{\begin{array}{l}
(10+15 \tau)\left(r_{l}^{L}(\tau)\right)^{2}+(50+15 \tau) r_{l}^{L}(\tau)=(80+15 \tau) \\
(40-15 \tau)\left(r_{t}^{L}(\tau)\right)^{2}+(80-15 \tau) r_{t}^{L}(\tau)=(120-15 \tau) \\
(5+10 \tau)\left(r_{l}^{U}(\tau)\right)^{2}+(45+10 \tau) r_{l}^{U}(\tau)=(75+10 \tau) \\
(45-10 \tau)\left(r_{t}^{U}(\tau)\right)^{2}+(95-20 \tau) r_{t}^{U}(\tau)=(125-10 \tau)
\end{array}\right.
$$

Table 1 clearly shows the dominance behavior of MM over $\mathrm{NN}$ in terms of absolute error on the same number of iterations $n=4$ for Example 1 .

Table 2 shows analytical solutions for Example 1.

Figure 5 shows initial guessed values, analytical, and numerical approximate solution graph of iterative methods $\mathrm{MM}$ and NN for interval-valued trapezoidal fuzzy nonlinear equation used in Example 1. 
To obtain initial guess, we use above system for $\tau=0$ and $\tau=1$; therefore,

$$
\begin{aligned}
& \left\{\begin{array}{l}
10\left(r_{l}^{L}\right)^{2}(0)+50 r_{l}^{L}(0)=80, \\
40\left(r_{t}^{L}\right)^{2}(0)+80 r_{t}^{L}(0)=120, \\
5\left(r_{l}^{U}\right)^{2}(0)+45 r_{l}^{U}(0)=75, \\
45\left(r_{t}^{U}\right)^{2}(0)+95 r_{t}^{U}(0)=125,
\end{array}\right. \\
& \left\{\begin{array}{l}
25\left(r_{l}^{L}\right)^{2}(1)+65 r_{l}^{L}(1)=95, \\
25\left(r_{t}^{L}\right)^{2}(1)+65 r_{t}^{L}(1)=105, \\
15\left(r_{l}^{U}\right)^{2}(1)+55 r_{l}^{U}(1)=85, \\
35\left(r_{t}^{U}\right)^{2}(1)+75 r_{t}^{U}(1)=115 .
\end{array}\right.
\end{aligned}
$$

Consequently, $r_{l}^{U}(0)=0.5, r_{l}^{L}(0)=0.5, r_{t}^{L}(0)=0.5, r_{t}^{U}(0$ )$=0.5$, and $r_{l}^{U}(0)=r_{l}^{L}(0)=r_{t}^{L}(0)=r_{t}^{U}(0)=1 / 2$. After 4 iterations, we obtain the solution with the maximum error less than $10^{-30}$. Now suppose $r$ is negative, we have the following:

$$
\left\{\begin{array}{l}
(10+15 \tau)\left(r_{l}^{L}(\tau)\right)^{2}+(50+15 \tau) r_{t}^{L}(\tau)=(80+15 \tau) \\
(40-15 \tau)\left(r_{t}^{L}(\tau)\right)^{2}+(80-15 \tau) r_{l}^{L}(\tau)=(120-15 \tau) \\
(5+10 \tau)\left(r_{l}^{U}(\tau)\right)^{2}+(45+10 \tau) r_{t}^{U}(\tau)=(75+10 \tau) \\
(45-10 \tau)\left(r_{t}^{U}(\tau)\right)^{2}+(95-20 \tau) r_{l}^{U}(\tau)=(125-10 \tau)
\end{array}\right.
$$

For $\tau=0$, we have $r_{l}^{L}(0)>r_{t}^{L}(0)$; therefore negative root does not exist.

Example 2. Consider the interval-valued trapezoidal fuzzy nonlinear equation

$$
\begin{aligned}
& \left\langle\left(0.65,0.73,0.87,0.95 ; \frac{2}{3}\right),(0.6,0.7,0.9,1 ; 1)\right\rangle(r(\tau))^{2} \\
& \quad+\left\langle\left(0.25,0.33,0.47,0.55 ; \frac{2}{3}\right),(0.2,0.3,0.5,0.6 ; 1)\right\rangle r(\tau) \\
& =\left\langle\left(0.45,0.53,0.67,0.75 ; \frac{2}{3}\right),(0.4,0.5,0.7,0.8 ; 1)\right\rangle .
\end{aligned}
$$

Without any loss of generality, assume that $r$ is positive; then, the parametric form of this equation is as follows:

$$
\begin{aligned}
\{ & \langle(0.65+0.12 \tau, 0.95-0.12 \tau),(0.6+0.1 \tau, 1-0.1 \tau)\rangle \\
& \cdot(r(\tau))^{2}+\langle(0.25+0.12 \tau, 0.55-0.12 \tau), \\
& (0.2+0.1 \tau, 0.6-0.1 \tau)\rangle r(\tau) \\
= & \langle(0.45+0.12 \tau, 0.75-0.12 \tau),(0.4+0.1 \tau, 0.8-0.1 \tau)\rangle,
\end{aligned}
$$

or

$$
\left\{\begin{array}{l}
(0.65+0.12 \tau)\left(r_{l}^{L}(\tau)\right)^{2}+(0.25+0.12 \tau) r_{l}^{L}(\tau)=(0.45+0.12 \tau) \\
(0.95-0.12 \tau)\left(r_{t}^{L}(\tau)\right)^{2}+(0.55-0.12 \tau) r_{t}^{L}(\tau)=(0.75-0.12 \tau) \\
(0.6+0.1 \tau)\left(r_{l}^{U}(\tau)\right)^{2}+(0.2+0.1 \tau) r_{l}^{U}(\tau)=(0.4+0.1 \tau) \\
(1-0.1 \tau)\left(r_{t}^{U}(\tau)\right)^{2}+(0.6-0.1 \tau) r_{t}^{U}(\tau)=(0.8-0.1 \tau)
\end{array}\right.
$$

Table 3 clearly shows the dominance behavior of MM over $\mathrm{NN}$ in terms of absolute error on the same number of iterations $n=4$ for Example 2.

Table 4 shows analytical solutions for Example 2.

Figure 6 shows initial guessed values, analytical, and numerical approximate solution graph of iterative methods $\mathrm{MM}$ and NN for interval-valued trapezoidal fuzzy nonlinear equation used in Example 2.

To obtain initial guess, we use above system for $\tau=0$ and $\tau=1$; therefore,

$$
\begin{aligned}
& \left\{\begin{array}{l}
0.65\left(r_{l}^{L}\right)^{2}(0)+0.2 r_{l}^{L}(0)=0.45, \\
0.95\left(r_{t}^{L}\right)^{2}(0)+0.55 r_{t}^{L}(0)=0.75, \\
0.6\left(r_{l}^{U}\right)^{2}(0)+0.2 r_{l}^{U}(0)=0.4, \\
1.0\left(r_{t}^{U}\right)^{2}(0)+0.6 r_{t}^{U}(0)=0.8,
\end{array}\right. \\
& \left\{\begin{array}{l}
0.77\left(r_{l}^{L}\right)^{2}(1)+0.37 r_{l}^{L}(1)=0.57, \\
0.83\left(r_{t}^{L}\right)^{2}(1)+0.43 r_{t}^{L}(1)=0.63, \\
0.7\left(r_{l}^{U}\right)^{2}(1)+0.3 r_{l}^{U}(1)=0.5, \\
0.9\left(r_{t}^{U}\right)^{2}(1)+0.5 r_{t}^{U}(1)=0.7 .
\end{array}\right.
\end{aligned}
$$

Consequently, $r_{l}^{U}(0)=0.6, r_{l}^{L}(0)=0.6, r_{t}^{L}(0)=0.6, r_{t}^{U}(0$ )$=0.6$, and $r_{l}^{U}(0)=r_{l}^{L}(0)=r_{t}^{L}(0)=r_{t}^{U}(0)=1 / 2$. After 4 iterations, we obtain the solution with the maximum error less than $10^{-30}$. Now suppose $r$ is negative, we have

$$
\left\{\begin{array}{l}
(0.65+0.12 \tau)\left(r_{l}^{L}(\tau)\right)^{2}+(0.25+0.12 \tau) r_{l}^{L}(\tau)=(0.45+0.12 \tau) \\
(0.95-0.12 \tau)\left(r_{t}^{L}(\tau)\right)^{2}+(0.55-0.12 \tau) r_{t}^{L}(\tau)=(0.75-0.12 \tau) \\
(0.6+0.1 \tau)\left(r_{l}^{U}(\tau)\right)^{2}+(0.2+0.1 \tau) r_{l}^{U}(\tau)=(0.4+0.1 \tau) \\
(1-0.1 \tau)\left(r_{t}^{U}(\tau)\right)^{2}+(0.6-0.1 \tau) r_{t}^{U}(\tau)=(0.8-0.1 \tau)
\end{array}\right.
$$

For $\tau=0$, we have $r_{l}^{L}(0)>r_{t}^{L}(0)$, therefore, negative root does not exist. 
Example 3. Consider the interval-valued trapezoidal fuzzy nonlinear equation

$$
\begin{aligned}
& \left\langle\left(0.45,0.53,0.67,0.75 ; \frac{2}{3}\right),(0.4,0.5,0.7,0.8 ; 1)\right\rangle(r(\tau))^{3} \\
& \quad+\left\langle\left(0.65,0.73,0.87,0.95 ; \frac{2}{3}\right),(0.6,0.7,0.9,1 ; 1)\right\rangle \sin (r(\tau)) \\
& =\left\langle\left(0.25,0.33,0.47,0.55 ; \frac{2}{3}\right),(0.2,0.3,0.5,0.6 ; 1)\right\rangle .
\end{aligned}
$$

Without any loss of generality, assume that $r$ is positive; then, the parametric form of this equation is as follows:

$$
\begin{aligned}
\{\langle & (0.45+0.12 \tau, 0.75-0.12 \tau),(0.4+0.1 \tau, 0.8-0.1 \tau)\rangle \\
& \cdot(r(\tau))^{3}+\langle(0.65+0.12 \tau, 0.95-0.12 \tau), \\
& (0.6+0.1 \tau, 1.0-0.1 \tau)\rangle r(\tau) \\
= & \langle(0.25+0.12 \tau, 0.55-0.12 \tau),(0.2+0.1 \tau, 0.6-0.1 \tau)\rangle,
\end{aligned}
$$

or

$$
\left\{\begin{array}{l}
(0.45+0.12 \tau) r_{l}^{L}(\tau)^{3}+(0.65+0.12 \tau) \sin \left(r_{l}^{L}(\tau)\right)=(0.25+0.12 \tau), \\
(0.75-0.12 \tau)\left(r_{t}^{L}(\tau)\right)^{3}+(0.95-0.12 \tau) \sin \left(r_{t}^{L}(\tau)\right)=(0.55-0.12 \tau), \\
(0.4+0.1 \tau)\left(r_{l}^{U}(\tau)\right)^{3}+(0.6+0.1 \tau) \sin \left(r_{l}^{U}(\tau)\right)=(0.2+0.1 \tau), \\
(0.8-0.1 \tau)\left(r_{t}^{U}(\tau)\right)^{3}+(1.0-0.1 \tau) \sin \left(r_{t}^{U}(\tau)\right)=(0.6-0.1 \tau) .
\end{array}\right.
$$

Table 5 clearly shows the dominance behavior of MM over NN in terms of absolute error on the same number of iterations $n=4$ for Example 3.

Table 6 shows analytical solutions for Example 3.

Figure 7 shows initial guessed values, analytical, and numerical approximate solution graph of iterative methods MM and NN for interval-valued trapezoidal fuzzy nonlinear equation used in Example 3.

To obtain initial guess, we use above system for $\tau=0$ and $\tau=1$; therefore,

$$
\begin{aligned}
& \left\{\begin{array}{l}
0.45\left(r_{l}^{L}\right)^{3}(0)+0.65 \sin \left(r_{l}^{L}(0)\right)=0.25 \\
0.75\left(r_{t}^{L}\right)^{3}(0)+0.95 \sin \left(r_{t}^{L}(0)\right)=0.55 \\
0.4\left(r_{l}^{U}\right)^{3}(0)+0.6 \sin \left(r_{l}^{U}(0)\right)=0.2, \\
0.8\left(r_{t}^{U}\right)^{3}(0)+1 \sin \left(r_{t}^{U}(0)\right)=0.6
\end{array}\right. \\
& \left\{\begin{array}{l}
0.57\left(r_{l}^{L}\right)^{3}(1)+0.77 \sin \left(r_{l}^{L}(1)\right)=0.37 \\
0.63\left(r_{t}^{L}\right)^{3}(1)+0.83 \sin \left(r_{t}^{L}(1)\right)=0.43, \\
0.5\left(r_{l}^{U}\right)^{3}(1)+0.7 \sin \left(r_{l}^{U}(1)\right)=0.3, \\
0.7\left(r_{t}^{U}\right)^{3}(1)+0.9 \sin \left(r_{t}^{U}(1)\right)=0.5 .
\end{array}\right.
\end{aligned}
$$

Consequently, $r_{l}^{U}(0)=0.5, r_{l}^{L}(0)=0.3, r_{t}^{L}(0)=0.5, r_{t}^{U}(0$ )$=0.3$, and $r_{l}^{U}(0)=r_{l}^{L}(0)=r_{t}^{L}(0)=r_{t}^{U}(0)=1 / 2$. After 4 iterations, we obtain the solution with the maximum error less than $10^{-30}$. Now suppose $r$ is negative, we have

$$
\left\{\begin{array}{l}
(0.45+0.12 \tau)\left(r_{l}^{L}(\tau)\right)^{3}+(0.65+0.12 \tau) \sin \left(r_{t}^{L}(\tau)\right)=(0.25+0.12 \tau), \\
(0.75-0.12 \tau)\left(r_{t}^{L}(\tau)\right)^{3}+(0.95-0.12 \tau) \sin \left(r_{l}^{L}(\tau)\right)=(0.55-0.12 \tau), \\
(0.4+0.1 \tau)\left(r_{l}^{U}(\tau)\right)^{3}+(0.6+0.1 \tau) \sin \left(r_{t}^{U}(\tau)\right)=(0.2+0.1 \tau), \\
(0.8-0.1 \tau)\left(r_{t}^{U}(\tau)\right)^{3}+(1.0-0.1 \tau) \sin \left(r_{l}^{U}(\tau)\right)=(0.6-0.1 \tau) .
\end{array}\right.
$$

For $\tau=0$, we have hence $r_{l}^{L}(0)>r_{t}^{L}(0)$; therefore, negative root does not exist.

Figures 8(a)-8(c) show residual falls for iterative methods $\mathrm{MM}$ and NN for interval-valued trapezoidal fuzzy nonlinear equation used in Examples 1-3, respectively.

\section{Conclusion}

In this research paper, we constructed highly efficient twostep numerical iterative method to approximate roots of interval-valued trapezoidal fuzzy nonlinear equations. A set of real-life applications from optimization are considered as a numerical test examples showing the practical performance and dominance efficiency of MM over NN method on the same number of iterations. From Tables 1-6 and Figures 18 , we observe that numerical results of MM methods are better in terms of absolute error and CPU time as compared to NN method. Considering the same ways as in this article, we can establish higher order and efficient numerical iterative methods for solving system of fuzzy nonlinear equations.

\section{Data Availability}

No data were used to support this study.

\section{Conflicts of Interest}

The authors declare that there are no conflicts of interest regarding the publication of this article.

\section{Authors' Contributions}

All authors contributed equally in the preparation of this manuscript.

\section{Acknowledgments}

The first author would like to thank the Deanship of Scientific Research at Majmaah University for supporting this work under Project Number R-2021-170.

\section{References}

[1] S. S. L. Chang and L. A. Zadeh, "On fuzzy mapping and control," IEEE Transactions on Systems, Man and Cybernetics, vol. 2, pp. 30-34, 1972. 
[2] D. Dubois and H. Prade, "Operations on fuzzy numbers," International Journal of Systems Science, vol. 9, no. 6, pp. 613-626, 1978.

[3] M. Mizumoto and K. Tanaka, "Some properties of fuzzy numbers," in Advances in fuzzy set theory and applications, pp. 153-164, North-Holland, Amsterdam-New York, 1979.

[4] M. Mizumoto and K. Tanaka, "The four operations of arithmetic on fuzzy numbers," Systems Computers and Controls, vol. 7, no. 5, pp. 73-81, 1976.

[5] M. Keyanpour and T. Akbarian, "Solving intuitionistic fuzzy nonlinear equations," Journal of Fuzzy set Value Analysis, vol. 2014, 2014.

[6] S. Nahmias, "Fuzzy variables," Fuzzy Sets and Systems, vol. 12, pp. 97-111, 1978.

[7] L. A. Zadeh, "Fuzzy sets," Information and Control, vol. 8, no. 3, pp. 338-353, 1965.

[8] L. A. Zadeh, "The concept of a linguistic variable and its application to approximation reasoning," Information Sciences, vol. 3, pp. 199-249, 1975.

[9] J. J. Buckley and Y. Qu, "Solving linear and quadratic fuzzy equations," Fuzzy Sets and Systems, vol. 38, no. 1, pp. 43-59, 1990.

[10] J. J. Buckley and Y. Qu, “On using $\alpha$-cuts to evaluate fuzzy equations," Fuzzy Sets and Systems, vol. 38, no. 3, pp. 309$312,1990$.

[11] J. J. Buckley and Y. Qu, "Solving fuzzy equations: a new solution concept," Fuzzy Sets and Systems, vol. 39, no. 3, pp. 291-301, 1991.

[12] J. J. Buckley and Y. Qu, "Solving systems of linear fuzzy equations," Fuzzy Sets and Systems, vol. 43, no. 1, pp. 33-43, 1991.

[13] S. Abbasbandy and B. Asady, "Newton's method for solving fuzzy nonlinear equations," Applied Mathematics and Computation, vol. 159, no. 2, pp. 349-356, 2004.

[14] T. Allahviranloo and S. Asari, "Numerical solution of fuzzy polynomial by Newton-Raphson method," Journal of Applied Mathematics, Islamic Azad University of Lahijan, vol. 7, no. 4, pp. 17-44, 2011.

[15] M. Mosleh, "Solution of dual fuzzy polynomial equations by modified Adomian decomposition method," Fuzzy Information and Engineering, vol. 5, no. 1, pp. 45-56, 2013.

[16] Y. J. Cho, N. J. Huang, and S. M. Kang, "Nonlinear equations for fuzzy mappings in probabilistic normed spaces," Fuzzy Sets and Systems, vol. 110, no. 1, pp. 115-122, 2000.

[17] J. E. Dennis and R. B. Schnabel, Numerical Methods for Unconstrained Optimization and Nonlinear Equations, PrenticeHall, Hoboken, NJ, USA, 1983.

[18] J. Fang, "On nonlinear equations for fuzzy mappings in probabilistic normed spaces," Fuzzy Sets and Systems, vol. 131, no. 3, pp. 357-364, 2002.

[19] R. Goetschel and W. Voxman, "Elementary fuzzy calculus," Fuzzy Sets and Systems, vol. 18, no. 1, pp. 31-43, 1986.

[20] I. M. Sulaiman, M. Mamat, M. Y. Waziri, M. A. Mohamed, and F. S. Mohamad, "Solving fuzzy nonlinear equation via Levenberg-Marquardt method," Far East Journal of Mathematical Science, vol. 103, pp. 1547-1558, 2018.

[21] J. Ma and G. Feng, "An approach to $H_{\infty}$ control of fuzzy dynamic systems," Fuzzy Sets and Systems, vol. 137, no. 3, pp. 367-386, 2003.

[22] A. Koam, M. Akram, G. Muhammad, and N. Hussain, "LU decomposition scheme for solving -polar fuzzy system of lin- ear equations," Mathematical Problems in Engineering, vol. 2020, 19 pages, 2020.

[23] M. Akram, G. Muhammad, A. N. A. Koam, and N. Hussain, "Iterative methods for solving a system of linear equation in bipolar fuzzy environment," Mathematics, vol. 7, no. 728, pp. 1-25, 2019.

[24] D. Dubois and H. Prade, Fuzzy Sets and Systems: Theory and Application, Academic Press, New York, NY, USA, 1980.

[25] H. J. Zimmermann, Fuzzy Sets Theory and Its Application, Kluwer Academic Press, Dordrecht, Netherlands, 1991.

[26] M. Saqib, M. Akram, and S. Bashir, "Certain efficient iterative methods for bipolar fuzzy system of linear equations," Journal of Intelligent \& Fuzzy Systems, vol. 39, no. 3, pp. 3971-3985, 2020.

[27] M. Akram, G. Muhammad, and T. Allahviranloo, "Bipolar fuzzy linear system of equations," Computational and Applied Mathematics, vol. 38, no. 69, pp. 1-29, 2019.

[28] J. Chiang, "Fuzzy linear programming based on statistical confidence interval and interval-valued fuzzy set," European Journal of Operational Research, vol. 129, no. 1, pp. 65-86, 2001.

[29] J. S. Yao and F. T. Lin, "Constructing a fuzzy flow-shop sequencing model based on statistical data," International Journal of Approximate Reasoning, vol. 29, no. 3, pp. 215234, 2002.

[30] S. H. Wei and S. M. Chen, "Fuzzy risk analysis based on interval-valued fuzzy numbers," Expert Systems with Applications, vol. 36, no. 2, pp. 2285-2299, 2009.

[31] B. Farhadinia, "Sensitivity analysis in interval-valued trapezoidal fuzzy number linear programming problems," Applied Mathematical Modelling, vol. 38, no. 1, pp. 50-62, 2014.

[32] S. Akram, M. Shams, N. Rafiq, and N. A. Mir, "On the stability of Weierstrass type method with King's correction for finding all roots of non-linear function with engineering application," Applied Mathematical Sciences, vol. 14, no. 10, pp. 461-473, 2020. 